\title{
Theory of noninteracting fermions and bosons in the canonical ensemble
}

\author{
Hatem Barghathi $\odot,{ }^{1,2}$ Jiangyong Yu, ${ }^{1}$ and Adrian Del Maestro $\oplus^{3,4,1}$ \\ ${ }^{1}$ Department of Physics, University of Vermont, Burlington, Vermont 05405, USA \\ ${ }^{2}$ Department of Physics, Missouri University of Science and Technology, Rolla, Missouri 65409, USA \\ ${ }^{3}$ Department of Physics and Astronomy, University of Tennessee, Knoxville, Tennessee 37996, USA \\ ${ }^{4}$ Min H. Kao Department of Electrical Engineering and Computer Science, University of Tennessee, Knoxville, Tennessee 37996, USA
}

(Received 10 August 2020; accepted 18 October 2020; published 9 November 2020)

\begin{abstract}
We present a self-contained theory for the exact calculation of particle number counting statistics of noninteracting indistinguishable particles in the canonical ensemble. This general framework introduces the concept of auxiliary partition functions, and represents a unification of previous distinct approaches with many known results appearing as direct consequences of the developed mathematical structure. In addition, we introduce a general decomposition of the correlations between occupation numbers in terms of the occupation numbers of individual energy levels, that is valid for both nondegenerate and degenerate spectra. To demonstrate the applicability of the theory in the presence of degeneracy, we compute energy-level correlations up to fourth order in a bosonic ring in the presence of a magnetic field.
\end{abstract}

DOI: 10.1103/PhysRevResearch.2.043206

\section{INTRODUCTION}

In quantum statistical physics, the analysis of a fixed number $N$ of indistinguishable particles is difficult, even in the noninteracting limit. In such a canonical ensemble, the constraint on the total number of particles gives rise to correlations between the occupation probability and the corresponding occupation numbers of the available energy levels. As a result, the canonical treatment of noninteracting fermions and bosons is in general avoided, and it is often completely absent in introductory texts addressing quantum statistical physics [1-3]. The standard approach is to relax the fixed $N$ constraint and instead describe the system using the grand canonical ensemble. While in most cases this approximation can be trusted in the large- $N$ limit, it can explicitly fail in the low-temperature regime [4,5]. The situation is even worse if the low-temperature system under study is mesoscopic, containing a relatively small number of particles.

Recent advances in ultracold atom experiments [6-15] are making such conditions the rule rather than the exception, where the interactions between particles are often ignored, especially in the fermionic case [16]. Thus an accurate statistical representation of these systems should be canonical, enforcing the existence of fixed $N$. In a different context, particle number conservation can reduce the amount of quantum information (entanglement) that can be extracted from a quantum state [17-25] and thus the fixed $N$ constraint requires a canonical treatment. This is reflected in the calculation of

Published by the American Physical Society under the terms of the Creative Commons Attribution 4.0 International license. Further distribution of this work must maintain attribution to the author(s) and the published article's title, journal citation, and DOI. the symmetry resolved entanglement which has recently been studied in a variety of physical systems [26-37]. In more general settings, the presence of conservation laws could demand a canonical treatment, as in the case of nuclear statistical models [38-56].

Given its pedagogical and now practical importance, as well as the long history of the problem of noninteracting quantum particles, the last 50 years has provided a host of results, varying from general recursive relations that govern the canonical partition functions and the corresponding occupation numbers [5,57-67], to approximate [4,68-72] and exact results for some special cases [73-81]. More recently, for the case of nondegenerate energy spectra, the exact decomposition of higher-order occupation number correlations in terms of the occupation numbers of individual bosonic and fermionic energy levels has been reported $[63,64]$.

In this paper, we present a unified framework for the calculation of physical observables in the canonical ensemble for $N$ fermions or bosons that is applicable to general noninteracting Hamiltonians that may contain degenerate energy levels. We present an analysis of the mathematical structure of bosonic and fermionic canonical partition functions in the noninteracting limit that leads to a set of recursion relations for exactly calculating the energy-level occupation probabilities and average occupation numbers. Using argument from linear algebra, we show how higher-order correlations between the occupation numbers can be factorized, allowing them to be obtained from the knowledge of the occupation numbers of the corresponding energy levels, a canonical generalization of Wick's theorem [82]. The key observation yielding simplification of calculations in the canonical ensemble is that occupation probabilities and occupation numbers can be expressed via auxiliary partition functions (APFs) - canonical fermionic or bosonic partition functions that correspond to a set of energy levels that are obtained from the full spectrum 
of the targeted system by making a subset of the energy levels degenerate (increasing its degeneracy if it is already degenerate) or alternatively by excluding it from the spectrum. Results obtained via auxiliary partition functions are validated by demonstrating that a number of previously reported formulas can be naturally recovered in a straightforward fashion within this framework.

In a noninteracting system, observables such as the average energy and magnetization can be obtained solely from the knowledge of average occupation numbers; however, the calculation of the corresponding statistical fluctuations in such quantities, i.e., specific heat and magnetic susceptibility, requires knowledge of the fluctuations in occupation numbers and (in the canonical ensemble) correlations between them. Therefore, the factorization of correlations between occupation numbers in terms of the average occupation numbers of individual energy levels provides a simplified approach to calculate quantities such as specific heat and magnetic susceptibility of a given system. Here, we highlight a method to compute such correlations by considering a degenerate system of a finite periodic chain of noninteracting bosons that is influenced by an external uniform magnetic field.

The auxiliary partition function approach presented here provides a set of tools that can be used to analyze experimental data in low-density atomic gases where the number of particles is fixed. The application of the physically relevant canonical ensemble can eliminate errors introduced via the grand canonical approximation (especially in the inferred temperature) and lead to a more accurate interpretation of experimental results, including an improved diagnosis of the role of weak interaction effects. It is hoped that the relative simplicity of the mathematical approach presented in this paper may encourage the inclusion of the interesting topic of the canonical treatment of Fermi and Bose gases in college-level textbooks.

In Sec. II we present the general formalism of the theory, where we introduce APFs and write occupation probability distributions, occupation numbers, and their correlations in terms of the APFs. In the same section, we also show how some of the previously known results can be recovered in a straightforward fashion. In Sec. III, considering fermionic and bosonic systems, we derive the decomposition of higher-order occupation number correlations into individual energy-level occupation numbers for nondegenerate and degenerate energy spectra, alike. To illustrate the applicability of the theory, in Sec. IV, we consider a bosonic ring in the presence of a magnetic field. We conclude in Sec. V.

\section{NONINTERACTING INDISTINGUISHABLE PARTICLES IN THE CANONICAL ENSEMBLE}

As the thermodynamic properties of a system of noninteracting particles are governed by the single-particle spectrum and the underlying particle statistics, we begin by considering the general one-particle spectrum $\epsilon_{i}$, with $i \in \mathcal{S}=$ $\{1,2, \ldots, M\}$. For an unbounded spectrum $M \rightarrow \infty$. In the canonical ensemble defined by fixing the total particle number $N$, the canonical partition function $Z_{N} \equiv Z_{N}(\mathcal{S})$ for $N$ indis- tinguishable particles is defined by

$$
Z_{N}=\sum_{\left.\boldsymbol{n}\right|_{N}} X\left(\left.\boldsymbol{n}\right|_{N}\right)
$$

where

$$
X\left(\left.\boldsymbol{n}\right|_{N}\right)=\prod_{i \in \mathcal{S}} e^{-\beta \epsilon_{i} n_{i}}
$$

are the Boltzmann factors at inverse temperature $\beta=1 / k_{\mathrm{B}} T$ and the components $\left\{n_{i}\right\}$ of the vector $\left.\boldsymbol{n}\right|_{N}=\left.\left(n_{1}, \ldots, n_{M}\right)\right|_{N}$ are the occupation numbers for the corresponding energy levels satisfying $\sum_{i \in \mathcal{S}} n_{i}=N$. The summation in Eq. (1) runs over all the possible occupation vectors $\left.\boldsymbol{n}\right|_{N}$ which, in addition to conserving the total number of particles $N$, obeys occupation limits for each of the energy levels $\epsilon_{i}: n_{i} \leqslant n_{i}^{\max }$, thus $N \leqslant N_{\max }=\sum_{i} n_{i}^{\max }$.

Consider the spectrum defined by $\mathcal{S}$ as the union of disjoint subsets (subspectra) $\mathcal{S}^{(1)}$ and $\mathcal{S}^{(2)}=\mathcal{S} \backslash S^{(1)}$, i.e., $\mathcal{S}=\mathcal{S}^{(1)} \cup$ $\mathcal{S}^{(2)}$. Under this decomposition the Boltzmann factors in $\mathcal{S}$ can be factorized as $X\left(\left.\boldsymbol{n}\right|_{N}\right)=X\left(\left.\boldsymbol{n}^{(1)}\right|_{k}\right) X\left(\left.\boldsymbol{n}^{(2)}\right|_{N-k}\right)$, where $\left.\boldsymbol{n}^{(1)}\right|_{k}$ and $\left.\boldsymbol{n}^{(2)}\right|_{N-k}$ represent occupation vectors of $k=\sum_{i \in \mathcal{S}^{(1)}} n_{i}^{(1)}$ and $N-k$ particles in $\mathcal{S}^{(1)}$ and $\mathcal{S}^{(2)}$, respectively. Summing $X\left(\left.\boldsymbol{n}\right|_{N}\right)$ over all possible $\left.\boldsymbol{n}^{(1)}\right|_{k}$ and $\left.\boldsymbol{n}^{(2)}\right|_{N-k}$ gives

$$
\sum_{\left.\boldsymbol{n}^{(1)}\right|_{k}} \sum_{\left.\boldsymbol{n}^{(2)}\right|_{N-k}} X\left(\left.\boldsymbol{n}\right|_{N}\right)=Z_{k}\left(\mathcal{S}^{(1)}\right) Z_{N-k}\left(\mathcal{S}^{(2)}\right),
$$

where we have introduced the auxiliary partition functions (APFs)

$$
\begin{aligned}
Z_{k}\left(\mathcal{S}^{(1)}\right) & =\sum_{\left.\boldsymbol{n}^{(1)}\right|_{k}} X\left(\left.\boldsymbol{n}^{(1)}\right|_{k}\right), \\
Z_{N-k}\left(\mathcal{S}^{(2)}\right) & =\sum_{\left.\boldsymbol{n}^{(2)}\right|_{N-k}} X\left(\left.\boldsymbol{n}^{(2)}\right|_{N-k}\right) .
\end{aligned}
$$

For Eqs. (4) and (5) to satisfy the restrictions imposed by the per-energy-level maximum occupancies $\left\{n_{i}^{\max }\right\}$ and the fixed $N, k$ must satisfy $\max \left(0, N-N_{2}^{\max }\right) \leqslant k \leqslant \min \left(N, N_{1}^{\max }\right)$, where $N_{1}^{\max }$ and $N_{2}^{\max }$ are the maximum numbers of particles allowed in $\mathcal{S}^{(1)}$ and $\mathcal{S}^{(2)}$, respectively. Additionally, any vector $\left.\boldsymbol{n}\right|_{N}$ under these constraints can be decomposed into two allowed vectors $\left.\boldsymbol{n}^{(1)}\right|_{k}$ and $\left.\boldsymbol{n}^{(2)}\right|_{N-k}$ and vice versa. Thus the sum in Eq. (1) can be similarly decomposed as $\sum_{\left.\boldsymbol{n}\right|_{N}} \equiv$ $\sum_{k=k_{\min }}^{k_{\max }} \sum_{\boldsymbol{n}^{(1)} \mid k} \sum_{\left.\boldsymbol{n}^{(2)}\right|_{N-k}}$ where $k_{\min }=\max \left(0, N-N_{\max }^{(2)}\right)$ and $k_{\max }=\min \left(N, N_{\max }^{(1)}\right)$, yielding the full partition function

$$
Z_{N} \equiv \sum_{k=k_{\min }}^{k_{\max }} Z_{k}\left(\mathcal{S}^{(1)}\right) Z_{N-k}\left(\mathcal{S}^{(2)}\right) .
$$

Employing the convention that $Z_{N}(\mathcal{S})=0$ whenever $N$ is negative, or when it exceeds the maximum number of particles set by $\mathcal{S}$, the limits in the above summation can be simplified to $\sum_{k=0}^{N}$. The above notation can be made more explicit by specifying the subset of levels that are not included in the partition function

$$
Z_{N}^{\backslash \mathcal{S}^{(1)}} \equiv Z_{N}\left(\mathcal{S} \backslash \mathcal{S}^{(1)}\right)
$$


and thus, for $\mathcal{S}^{(1)}=\left\{j_{1}, j_{2}, \ldots, j_{\ell}\right\}$ containing $\ell$ levels, Eq. (6) is equivalent to

$$
Z_{N} \equiv \sum_{k=0}^{N} Z_{k}\left(\left\{j_{1}, j_{2}, \ldots, j_{\ell}\right\}\right) Z_{N-k}^{\backslash\left\{j_{1}, j_{2}, \ldots, j_{\ell}\right\}} .
$$

To obtain a physical interpretation of Eq. (8), recall that in the canonical ensemble the likelihood of the $N$-particle system being in a microstate defined by the occupation vector $\left.\boldsymbol{n}\right|_{N}$ is given by the ratio $X\left(\left.\boldsymbol{n}\right|_{N}\right) / Z_{N}$. Accordingly, for the subset of energy levels with indices $\left\{j_{1}, j_{2}, \ldots, j_{\ell}\right\}$, the joint probability distribution of the corresponding occupation numbers $\mathcal{P}_{n_{j_{1}}, n_{j_{2}}, \ldots, n_{j_{\ell}}}$ can be obtained by performing the summation $\sum_{\left.\boldsymbol{n}^{(2)}\right|_{N-k}}$, where $k=\sum_{r=1}^{\ell} n_{j_{r}}$, yielding

$$
\mathcal{P}_{n_{j_{1}}, n_{j_{2}}, \ldots, n_{j_{\ell}}}=\frac{e^{-\beta \sum_{r=1}^{\ell} \epsilon_{j_{r}} n_{j_{r}}}}{Z_{N}} Z_{N-k}^{\backslash\left\{j_{1}, j_{2}, \ldots, j_{\ell}\right\}} .
$$

It follows that the probability $P_{k}\left(\left\{j_{1}, j_{2}, \ldots, j_{\ell}\right\}\right)$ of finding $k$ particles in $\left\{j_{1}, j_{2}, \ldots, j_{\ell}\right\}$ and, of course, $N-k$ particles in $\mathcal{S} \backslash\left\{j_{1}, j_{2}, \ldots, j_{\ell}\right\}$ can be obtained by applying the summation $\sum_{\left.n^{(1)}\right|_{k}}$ :

$$
P_{k}\left(\left\{j_{1}, j_{2}, \ldots, j_{\ell}\right\}\right)=\frac{Z_{k}\left(\left\{j_{1}, j_{2}, \ldots, j_{\ell}\right\}\right) Z_{N-k}^{\backslash\left\{j_{1}, j_{2}, \ldots, j_{\ell}\right\}}}{Z_{N}},
$$

where the normalization of $P_{k}\left(\left\{j_{1}, j_{2}, \ldots, j_{\ell}\right\}\right)$ is guaranteed by Eq. (8). Note: The considered spectra $\mathcal{S}$ and its subsets could include degenerate levels, despite the distinct indices used to label the energy levels.

So far the analysis of the partition functions has been completely general and we have not specified what type of particles are being described. Now, let us be more specific and consider a system that solely consists of either fermions or bosons.

\section{A. Inverted analogy between fermionic and bosonic statistics}

Having developed an intuition for the general structure of the canonical partition function under a bipartition into subspectra, we now observe how this can provide insights into the relationship between fermionic $\left(n_{i}^{\max }=1\right)$ and bosonic $\left(n_{i}^{\max } \rightarrow \infty\right)$ statistics of $N$ noninteracting particles. To distinguish the two cases we introduce a new subscript on the partition function ( $F$ for fermions and $B$ for bosons).

If the set $\mathcal{S}^{(1)}$ represents a single energy level with an index $j_{1}=j$ then using our convention we have

$$
Z_{F, k}(\{j\})=\left\{\begin{array}{cc}
e^{-\beta \epsilon_{j} k} & 0 \leqslant k \leqslant 1 \\
0 & \text { otherwise }
\end{array}\right.
$$

for fermions and

$$
Z_{B, k}(\{j\})=\left\{\begin{array}{cc}
e^{-\beta \epsilon_{j} k} & k \geqslant 0 \\
0 & \text { otherwise }
\end{array}\right.
$$

for bosons. Substituting into Eq. (8) we immediately find

$$
\begin{gathered}
Z_{F, N}=Z_{F, N}^{\backslash\{j\}}+e^{-\beta \epsilon_{j}} Z_{F, N-1}^{\backslash\{j\}}, \\
Z_{B, N}=\sum_{k=0}^{N} e^{-\beta \epsilon_{j} k} Z_{B, N-k}^{\backslash\{j\}} .
\end{gathered}
$$

The relations in Eqs. (13) and (14) formally describe the procedure for generating the canonical partition function of the $N$-particle system after introducing an energy level $\epsilon_{j}$ to the preexisting spectrum $\mathcal{S} \backslash\{j\}$.

Examining the structure of Eq. (13) suggests a simple matrix form: $\boldsymbol{Z}=\mathrm{A} \boldsymbol{Z}^{\backslash\{j\}}$, where $\boldsymbol{Z}=\left(Z_{F, 0}, Z_{F, 1}, \ldots\right), \boldsymbol{Z}^{\backslash\{j\}}=$ $\left(Z_{F, 0}^{\backslash\{j\}}, Z_{F, 1}^{\backslash\{j\}}, \ldots\right)$, and the matrix $\mathrm{A}_{n, m}=\delta_{n, m}+e^{-\beta \epsilon_{j}} \delta_{n, m+1}$ is bidiagonal and can be inverted such that

$$
Z_{F, N}^{\backslash\{j\}}=\sum_{k=0}^{N}(-1)^{k} e^{-\beta \epsilon_{j} k} Z_{F, N-k} .
$$

Comparing this expression with Eq. (14), we observe an identical structure apart from exchanging the factor $e^{-\beta \epsilon_{j}}$ with $\left(-e^{-\beta \epsilon_{j}}\right)$. Thus we can obtain the inversion of Eq. (14) by replacing $e^{-\beta \epsilon_{j}}$ with $\left(-e^{-\beta \epsilon_{j}}\right)$ in Eq. (13), i.e.,

$$
Z_{B, N}^{\backslash\{j\}}=Z_{B, N}-e^{-\beta \epsilon_{j}} Z_{B, N-1} .
$$

The relations Eqs. (15) and (16) exemplify the elimination of an energy level as they represent the inverse of Eqs. (13) and (14), respectively.

If we absorb the negative signs in Eqs. (15) by shifting the energy $\epsilon_{j}$ by $\pm i \pi / \beta$, we can write $Z_{F, N}^{\backslash\{j\}}=\sum_{k=0}^{N} e^{-\beta \epsilon_{j}^{\prime} k} Z_{F, N-k}$, where $\epsilon_{j}^{\prime}=\epsilon_{j} \pm i \pi / \beta$. As the general bipartition into subspectra introduced in Eq. (6) holds for energy levels with mixed statistics, and does not require real entries for the $\epsilon_{i}$, we can build the bosonic partition functions $Z_{B N}^{\prime}\left(\left\{j_{1}, j_{2}, \ldots, j_{\ell}\right\}\right)$ using the shifted energies $\left\{\epsilon_{j_{1}}^{\prime}, \epsilon_{j_{2}}^{\prime}, \ldots, \epsilon_{j_{\ell}}^{\prime}\right\}$ and then combine them with the $Z_{F, N}$ to generate a mixed form:

$$
Z_{F, N}^{\left.\backslash j_{1}, j_{2}, \ldots, j_{\ell}\right\}}=\sum_{k=0}^{N} Z_{B, k}^{\prime}\left(\left\{j_{1}, j_{2}, \ldots, j_{\ell}\right\}\right) Z_{F, N-k} .
$$

This expression can also be validated using Eqs. (14) and (15) with a proof included in Appendix A. The effect of shifting the single-particle spectrum by a constant $\omega$ on the canonical partition function $Z_{N}$ is captured by a rescaling factor $e^{-\beta \omega N}$ and the resulting $N$-particle partition function of the shifted spectrum is $e^{-\beta \omega N} Z_{N}$. Using $Z_{B, k}^{\prime}\left(\left\{j_{1}, j_{2}, \ldots, j_{\ell}\right\}\right)=$ $e^{ \pm i \pi k} Z_{B, k}\left(\left\{j_{1}, j_{2}, \ldots, j_{\ell}\right\}\right)$ then yields

$$
Z_{F, N}^{\left.\backslash j_{1}, j_{2}, \ldots, j_{\ell}\right\}}=\sum_{k=0}^{N}(-1)^{k} Z_{B, k}\left(\left\{j_{1}, j_{2}, \ldots, j_{\ell}\right\}\right) Z_{F, N-k} .
$$

Starting from Eq. (16) and following the same argument, we obtain an equivalent expression for bosons:

$$
Z_{B, N}^{\left.\backslash j_{1}, j_{2}, \ldots, j_{\ell}\right\}}=\sum_{k=0}^{N}(-1)^{k} Z_{F, k}\left(\left\{j_{1}, j_{2}, \ldots, j_{\ell}\right\}\right) Z_{B, N-k} .
$$

The last two equations can be seen as a generalization of Eqs. (15) and (16). However, they also recover the symmetry between fermionic and bosonic statistics. To this end, we show how to obtain the partition function of a given spectrum using the APFs of two complementary subsets of the spectrum through Eq. (8). Also, Eqs. (18) and (19) show that this relation can be inverted, i.e., we can calculate the APFs of a subset of energy level using the APFs of its complement with the opposite statistics and the partition functions of the full spectrum. 


\section{B. Energy-level occupations and correlations}

For fermions, the Pauli exclusion principle restricts the number of particles occupying an energy level $\epsilon_{j}$ to $n_{j}=0$ or 1 . Equivalently, $n_{j}^{p}=n_{j}$ for any $p>0$, simplifying the calculation of energy-level occupation numbers and the correlations between them, including higher moments. More specifically, the average $\left\langle n_{j_{1}}^{p_{1}} n_{j_{2}}^{p_{2}} \ldots n_{j_{\ell}}^{p_{\ell}}\right\rangle_{F, N}=\left\langle n_{j_{1}} n_{j_{2}} \ldots n_{j_{\ell}}\right\rangle_{F, N}$, where $p_{i}>0, i \in\{1, \ldots, \ell\}$, and in general

$$
\left\langle n_{j_{1}} n_{j_{2}} \ldots n_{j_{\ell}}\right\rangle_{F, N}=\sum_{\boldsymbol{n}\left(\left\{j_{1}, j_{2}, \ldots, j_{\ell}\right\}\right)} n_{j_{1}} n_{j_{2}} \ldots n_{j_{\ell}} \mathcal{P}_{F, n_{j_{1}}, n_{j_{2}}, \ldots, n_{j_{\ell}}}
$$

using the probability

$$
\mathcal{P}_{F, n_{j_{1}}, n_{j_{2}}, \ldots, n_{j_{\ell}}}=\frac{e^{-\beta \sum_{r=1}^{\ell} \epsilon_{j_{r}} n_{j_{r}}}}{Z_{F, N}} Z_{F, N-\sum_{r=1}^{\ell} n_{j_{r}}}^{\left.\backslash j_{1}, j_{2}, \ldots, j_{\ell}\right\}},
$$

defined in Eq. (9). The only term that survives in Eq. (20) has $n_{j_{1}}=n_{j_{2}}=\cdots=n_{j_{\ell}}=1$, giving

$$
\left\langle n_{j_{1}} n_{j_{2}} \ldots n_{j_{\ell}}\right\rangle_{F, N}=\frac{e^{-\beta \sum_{r=1}^{\ell} \epsilon_{j r}}}{Z_{F, N}} Z_{F, N-\ell}^{\backslash\left\{j_{1}, j_{2}, \ldots, j_{\ell}\right\}} .
$$

Following the same procedure, Eq. (20) can be generalized to describe both correlations and anticorrelations between energy levels:

$$
\begin{gathered}
\left\langle\prod_{r=1}^{\ell}\left[n_{j_{r}} \gamma_{j_{r}}+\left(1-n_{j_{r}}\right)\left(1-\gamma_{j_{r}}\right)\right]\right\rangle_{F, N} \\
=\frac{1}{Z_{F, N}} e^{-\beta \sum_{r=1}^{\ell} \epsilon_{j_{r}} \gamma_{j_{r}}} Z_{F, N-\sum_{r=1}^{\ell} \gamma_{j_{r}}}^{\backslash\left\{j_{1}, j_{2}, \ldots, j_{\ell}\right\}},
\end{gathered}
$$

where $\gamma_{j_{r}}=1,0$. For the latter with $\gamma_{j_{r}}=0$, the occupation numbers in Eq. (20) have been replaced with their complements, $1-n_{j_{r}}$. Thus, we find that fermionic level occupations and correlations can be directly written in terms of APFs without resorting to the usual definition $\left\langle n_{j_{1}} n_{j_{2}} \ldots n_{j_{\ell}}\right\rangle_{F, N}=$ $\frac{1}{Z_{F, N}} \frac{\partial^{\ell} Z_{F, N}}{\partial\left(-\beta \epsilon_{j_{1}}\right) \ldots \partial\left(-\beta \epsilon_{j_{\ell}}\right)}$ which yields equivalent results.

When considering a single level $(\ell=1)$, the occupation probability of the $j$ th fermionic level immediately follows:

$$
\left\langle n_{j}\right\rangle_{F, N}=\frac{e^{-\beta \epsilon_{j}} Z_{F, N-1}^{\backslash\{j\}}}{Z_{F, N}},
$$

with the associated probability

$$
\mathcal{P}_{F, n_{j}}=\frac{e^{-\beta \epsilon_{j} n_{j}}}{Z_{F, N}} Z_{F, N-n_{j}}^{\backslash\{j\}} .
$$

For bosons, the occupation numbers $\left\langle n_{j}\right\rangle_{B, N}$ can be calculated from the corresponding occupation probability distribution $\mathcal{P}_{n_{j}}$ which is obtained from Eq. (9):

$$
\mathcal{P}_{B, n_{j}}=\frac{e^{-\beta \epsilon_{j} n_{j}}}{Z_{B, N}} Z_{B, N-n_{j}}^{\backslash\{j\}},
$$

and for the $\ell$-point correlations

$$
\mathcal{P}_{B, n_{j_{1}}, n_{j_{2}}, \ldots, n_{j_{\ell}}}=\frac{e^{-\beta \sum_{r=1}^{\ell} \epsilon_{j_{r}} n_{j_{r}}}}{Z_{B, N}} Z_{B, N-\sum_{r=1}^{\ell} n_{j_{r}}}^{\left.\backslash j_{1}, j_{2}, \ldots, j_{\ell}\right\}} .
$$

However, unlike the fermionic case, such an approach requires performing the unrestricted summation $\sum_{\boldsymbol{n}\left(\left\{j_{1}, j_{2}, \ldots, j_{\ell}\right\}\right)}$.
An alternative method which avoids this difficulty can be developed by exploiting the inverted analogy between fermionic and bosonic statistics introduced in Sec. II A. In the fermionic case, the occupation number of an energy level $\epsilon_{j}$ is proportional to the APF $Z_{F, N-1}^{\backslash\{j\}}$ [Eq. (24)] which corresponds to the actual spectrum of the system missing the energy level $\epsilon_{j}$. This suggests a route forward for bosons via the analogous inversion of doubly including the energy level $\epsilon_{j}$ instead of removing it, i.e., we construct an APF where this level is twofold degenerate. We denote the corresponding $N$-boson APF by $Z_{B, N}^{\cup\{j\}}$ and distinguish the two levels using the dressed indices $j^{(0)}$ and $j^{(1)}$ such that the resulting combined spectrum has level indices $\left\{1, \ldots j-1, j^{(0)}, j^{(1)}, j+1, \ldots, M\right\}$ where $\epsilon_{j}=\epsilon_{j^{(0)}}=\epsilon_{j^{(1)}}$.

Returning to the general definition of the canonical partition function in Eq. (1), we can write $Z_{B, N}^{\cup\{j\}}=\sum_{\boldsymbol{n}^{\prime} \mid N} X\left(\left.\boldsymbol{n}^{\prime}\right|_{N}\right)$, where the occupation vectors $\left.\boldsymbol{n}^{\prime}\right|_{N}$ have one extra component: $n_{j}$ in $\left.\boldsymbol{n}\right|_{N}$ is replaced by $n_{j^{(0)}}$ and $n_{j^{(1)}}$. The modified Boltzmann factors are

$$
\left.X\left(\left.\boldsymbol{n}^{\prime}\right|_{N}\right)=e^{-\beta \epsilon_{j}\left[n_{j}(0)+n_{j}(1)\right.}\right] \prod_{i \neq j^{(0)}, j^{(1)}} e^{-\beta \epsilon_{i} n_{i}}
$$

and thus their value is dependent only on the total occupancy of the $j$ th level, $n_{j^{(0)}}+n_{j^{(1)}}$. As a result, $X\left(\left.\boldsymbol{n}\right|_{N}\right)=X\left(\left.\boldsymbol{n}^{\prime}\right|_{N}\right)$ for any occupation vectors $\left.\boldsymbol{n}\right|_{N}$ and $\left.\boldsymbol{n}^{\prime}\right|_{N}$ with all $i \neq j$ components equal as well as $\left[\left.\boldsymbol{n}\right|_{N}\right]_{j}=n_{j}=n_{j^{(0)}}+n_{j^{(1)}}$. For fixed $\left.\boldsymbol{n}\right|_{N}$, the number of vectors $\left.\boldsymbol{n}^{\prime}\right|_{N}$ that satisfies the previous conditions is equal to $n_{j}+1$, or the number of ways in which $n_{j}$ bosons can occupy two energy levels. The APF can then be written in terms of the original occupation vector $\left.\boldsymbol{n}\right|_{N}$ by inserting a frequency factor to account for the extra level $j$ :

$$
\begin{aligned}
Z_{B, N}^{\cup\{j\}} & =\sum_{\left.\boldsymbol{n}\right|_{N}}\left(n_{j}+1\right) X\left(\left.\boldsymbol{n}\right|_{N}\right) \\
& =Z_{B, N}\left\langle n_{j}+1\right\rangle_{B, N}
\end{aligned}
$$

and thus we can write

$$
\left\langle n_{j}\right\rangle_{B, N}=Z_{B, N}^{\cup\{j\}} / Z_{B, N}-1 .
$$

Applying Eq. (16) gives $Z_{B, N}^{\cup\{j\}}=Z_{B, N}+e^{-\beta \epsilon_{j}} Z_{B, N-1}^{\cup\{j\}}$, which can be substituted into Eq. (30) to arrive at

$$
\left\langle n_{j}\right\rangle_{B, N}=\frac{e^{-\beta \epsilon_{j}} Z_{B, N-1}^{\cup\{j\}}}{Z_{B, N}},
$$

which is in the same form as Eq. (24) for fermions.

To generalize this expression to $\ell$-level correlations with $\ell>1$ we examine the numerator of Eq. (31), recalling that we have added an extra copy of energy level $j$ to the partition function for $N-1$ particles such that it now appears $m_{j}+$ $1=2$ times in the associated spectrum where, in general, $m_{j}$ is the number of the added copies of energy level $j$ :

$$
\begin{aligned}
e^{-\beta \epsilon_{j}} Z_{B, N-1}^{\cup\{j\}} & =e^{-\beta \epsilon_{j}} \sum_{\left.\boldsymbol{n}^{\prime}\right|_{N-1}} X\left(\left.\boldsymbol{n}^{\prime}\right|_{N-1}\right) \\
& =\sum_{\left.\boldsymbol{n}\right|_{N-1}}\left(\begin{array}{c}
\tilde{n}_{j}+\left(m_{j}+1\right)-1 \\
\left(m_{j}+1\right)-1
\end{array}\right) e^{-\beta \epsilon_{j}} X\left(\left.\boldsymbol{n}\right|_{N-1}\right) \\
& =\sum_{\left.\boldsymbol{n}\right|_{N, n_{j} \geqslant 1}} n_{j} X\left(\left.\boldsymbol{n}\right|_{N}\right) .
\end{aligned}
$$


Here we obtain the second line using the same trick as in Eq. (29) to convert $X\left(\left.\boldsymbol{n}^{\prime}\right|_{N-1}\right)$ into $X\left(\left.\boldsymbol{n}\right|_{N-1}\right)$ by accounting for the degeneracy where $\left[\left.\boldsymbol{n}\right|_{N-1}\right]_{j}=\tilde{n}_{j} \geqslant 0$ is the total number of particles occupying the level $j$ as it appears in the Boltzmann factor $X\left(\left.\boldsymbol{n}\right|_{N-1}\right)=e^{-\beta \epsilon_{j} \tilde{n}_{j}} \prod_{i \neq j} e^{-\beta \epsilon_{i} n_{i}}$. The complicated looking binomial coefficient $\left(\begin{array}{c}\tilde{n}_{j}+\left(m_{j}+1\right)-1 \\ \left(m_{j}+1\right)-1\end{array}\right)=\left(\left(\begin{array}{c}\tilde{n}_{j} \\ m_{j}+1\end{array}\right)\right)$ is the multiset coefficient that counts the number of ways $\tilde{n}_{j}$ bosons can be distributed amongst the $m_{j}+1$ levels with energy $\epsilon_{j}$. Finally, the last line is obtained by using the fact that $e^{-\beta \epsilon_{j}} X\left(\left.\boldsymbol{n}\right|_{N-1}\right)=X\left(\left.\boldsymbol{n}\right|_{N}\right)$ where $\left[\left.\boldsymbol{n}\right|_{N}\right]_{j}=n_{j}=\tilde{n}_{j}+1 \geqslant 1$.

Equation (32) can be immediately extended to the case where we add copies of not one but $\ell$ levels $\left\{j_{1}, \ldots, j_{\ell}\right\}$ :

$$
e^{-\beta \sum_{r=1}^{\ell} \epsilon_{j_{r}}} Z_{B, N-\ell}^{\cup\left\{j_{1}, \ldots, j_{\ell}\right\}}=\sum_{\left.\boldsymbol{n}\right|_{N}}\left(\prod_{r=1}^{\ell} n_{j_{r}}\right) X\left(\left.\boldsymbol{n}\right|_{N}\right)
$$

where the conditions $n_{j_{r}} \geqslant 1$ in the occupancy vector can be neglected as any $n_{j_{r}}=0$ terms do not contribute to the sum due to the multiplicative string. Finally, we can write the desired result:

$$
\left\langle n_{j_{1}} n_{j_{2}} \ldots n_{j_{\ell}}\right\rangle_{B, N}=\frac{e^{-\beta \sum_{r=1}^{\ell} \epsilon_{j_{r}}}}{Z_{B, N}} Z_{B, N-\ell}^{\cup\left\{j_{1}, j_{2}, \ldots, j_{\ell}\right\}} .
$$

An immediate extension of Eq. (34) will turn out to be useful, which introduces an APF with higher-order degeneracy. We consider $m_{j_{r}}$ extra copies of the $r$ th level $\epsilon_{j_{r}}$ with $r \in\{1, \ldots \ell\}$ and find

$$
\begin{aligned}
& \left\langle\prod_{r=1}^{\ell}\left(\begin{array}{c}
n_{j_{r}}-q_{j_{r}}+m_{j_{r}} \\
m_{j_{r}}
\end{array}\right)\right\rangle_{B, N} \\
& \quad=\frac{1}{Z_{B, N}} e^{-\beta \sum_{r=1}^{\ell} \epsilon_{j_{r}} q_{j_{r}}} Z_{B, N-\sum_{r=1}^{\ell} q_{j_{r}}}^{\cup\left\{j_{1}^{(1)}, \ldots, j_{1}^{\left(m_{j_{1}}\right)}, \ldots j_{\ell}^{(1)}, \ldots, j_{\ell}^{\left(m_{j_{\ell}}\right)}\right\}}
\end{aligned}
$$

where the $q_{j_{r}} \leqslant m_{j_{r}}$ allow for the added freedom of choice of how many particles are associated with each of the degenerate levels and allow us to write the left-hand side in terms of the desired occupations $n_{j}=\left[\left.\boldsymbol{n}\right|_{N}\right]_{j}$. Note: To simplify notation we only include a superscript on the levels in the bosonic $\mathrm{APF}$ and only if we are adding more than one extra copy per original level.

Equations (23) and (35) are the major results of this section, and demonstrate that for both fermions and bosons $\ell$-level correlations can be written in terms of APFs for $N-\ell$ particles with $\ell$ energy levels removed (added) for fermions (bosons).

\section{Recovering known results via the APF theory}

In this section, we illustrate the utility of our auxiliary expressions in simplifying the derivation of known recursion relations that govern the canonical partition functions and occupation numbers for fermions and bosons.

Beginning with fermionic statistics, if we use the definition of $\left\langle n_{j}\right\rangle_{F, N}$ in Eq. (24) to substitute for $Z_{F, N}^{\backslash\{j\}}$ and $Z_{F, N-1}^{\backslash\{j\}}$ in Eq. (13), we obtain the well-known recursion relation for occupation numbers $[57,59]$ :

$$
\left\langle n_{j}\right\rangle_{F, N+1}=\frac{Z_{F, N}}{Z_{F, N+1}} e^{-\beta \epsilon_{j}}\left(1-\left\langle n_{j}\right\rangle_{F, N}\right) .
$$

$\left\langle n_{j}\right\rangle_{F, N}$ can be written explicitly in terms of the partition functions by substituting for $Z_{F, N-1}^{\backslash\{j\}}$ in Eq. (24) using Eq. (15) as

$$
\left\langle n_{j}\right\rangle_{F, N}=\frac{1}{Z_{F, N}} \sum_{k=1}^{N}(-1)^{k-1} e^{-\beta \epsilon_{j} k} Z_{F, N-k},
$$

and using the canonical condition $\sum_{i}\left\langle n_{i}\right\rangle_{F, N}=N$ we find the original 1993 result of Borrmann and Franke [58]:

$$
Z_{F, N}=\frac{1}{N} \sum_{k=1}^{N}(-1)^{k-1} C_{k} Z_{F, N-k},
$$

where $C_{k}=\sum_{j} e^{-\beta \epsilon_{j} k}$.

Bosonic statistics can be treated in analogy to the fermionic case. Assigning the roles played by Eqs. (24), (13), and (15) to Eqs. (31), (16), and (14) [83], respectively, we obtain the known bosonic equivalents [57-59]:

$$
\begin{aligned}
\left\langle n_{j}\right\rangle_{B, N+1} & =\frac{Z_{B, N}}{Z_{B, N+1}} e^{-\beta \epsilon_{j}}\left(1+\left\langle n_{j}\right\rangle_{B, N}\right), \\
\left\langle n_{j}\right\rangle_{B, N} & =\frac{1}{Z_{B, N}} \sum_{k=1}^{N} e^{-\beta \epsilon_{j} k} Z_{B, N-k}, \\
Z_{B, N} & =\frac{1}{N} \sum_{k=1}^{N} C_{k} Z_{B, N-k} .
\end{aligned}
$$

Due to the Pauli exclusion principle for fermionic statistics, the occupation number $\left\langle n_{j}\right\rangle_{F, N}$ of an energy level $j$ gives us direct access to the occupation probability distribution $\mathcal{P}_{F, n_{j}}$ of the level. Despite the absence of any such simplification in the bosonic case, the occupation probability distribution $\mathcal{P}_{B, n_{j}}$ of a single energy level can be related to the corresponding partition functions as

$$
\mathcal{P}_{B, n_{j}}=e^{-\beta \epsilon_{j} n_{j}} \frac{Z_{B, N-n_{j}}}{Z_{B, N}}-e^{-\beta \epsilon_{j}\left(n_{j}+1\right)} \frac{Z_{B, N-n_{j}-1}}{Z_{B, N}},
$$

which is obtained by using Eq. (26) to substitute for $Z_{B, N-n_{j}}^{\backslash\{j\}}$ in Eq. (16), after replacing $N$ with $N-n_{j}$ [61]. In summary, within the unified framework of APFs, it is straightforward to obtain most of the well-known general relations in the fermionic and bosonic canonical ensemble that were previously derived using a host of different methods. This highlights the utility of this approach as a unifying framework when studying $N$ indistinguishable noninteracting particles.

\section{General expressions for probabilities and correlations}

De facto, the APFs can also be used to generalize previous results, and in a form that is highly symmetric with respect to particle statistics. To accentuate this, let us introduce the notation

$$
\zeta= \begin{cases}+1 & \Leftrightarrow B \Leftrightarrow \text { bosons } \\ -1 & \Leftrightarrow F \Leftrightarrow \text { fermions }\end{cases}
$$

Then, the recursive relations for energy-level correlations can be obtained using Eqs. (13) and (16) for fermions and bosons, respectively. The number of initial values of correlations needed is equal to the number of the involved points; e.g., for 
the two-level correlations we find

$$
\begin{aligned}
\left\langle n_{i} n_{j}\right\rangle_{\zeta, N+2}= & \frac{Z_{\zeta, N}}{Z_{\zeta, N+2}} e^{-\beta\left(\epsilon_{i}+\epsilon_{j}\right)}\left(1-\left\langle n_{i} n_{j}\right\rangle_{\zeta, N}\right) \\
& +\zeta \frac{Z_{\zeta, N+1}}{Z_{\zeta, N+2}}\left(e^{-\beta \epsilon_{i}}+e^{-\beta \epsilon_{j}}\right)\left\langle n_{i} n_{j}\right\rangle_{\zeta, N+1} .
\end{aligned}
$$

Further, for the set of levels $\mathcal{S}_{\ell}=\left\{j_{1}, \ldots, j_{\ell}\right\}$, Eqs. (21) and (27), define the joint probability distributions of the occupation numbers in terms of the APFs $Z_{F, k}^{\backslash \mathcal{S}_{\ell}}$ and $Z_{B, k}^{\backslash \mathcal{S}_{\ell}}$, which can be reexpressed using Eqs. (18) and (19) as

$$
\mathcal{P}_{\zeta, n_{j_{1}}, \ldots, n_{j_{\ell}}}=\frac{e^{-\beta E_{\mathrm{tot}}}}{Z_{\zeta, N}} \sum_{k=0}^{N-n_{\mathrm{tot}}}(-1)^{k} Z_{\zeta, k}\left(\mathcal{S}_{\ell}\right) Z_{\zeta, N-n_{\mathrm{tot}}-k}
$$

where $E_{\mathrm{tot}}=\sum_{r=1}^{\ell} \epsilon_{j_{r}} n_{j_{r}}$ and $n_{\mathrm{tot}}=\sum_{r=1}^{\ell} n_{j_{r}}$. To avoid confusion we note again the convention in use that $Z_{\zeta, k}\left(\mathcal{S}_{\ell}\right)=0$ whenever $k$ exceeds the maximum number of particles set by $\mathcal{S}_{\ell}$ for fixed particle statistics.

Now, as the level occupation correlations of fermions and bosons are represented by the APFs $Z_{F, k}^{\backslash \mathcal{S}_{\ell}}$ and $Z_{B, k}^{\cup \mathcal{S}_{\ell}}$ in Eqs. (22) and (34), we can write

$$
\left\langle n_{j_{1}} \ldots n_{j_{\ell}}\right\rangle_{\zeta, N}=\frac{e^{-\beta \sum_{r=1}^{\ell} \epsilon_{j r}}}{Z_{\zeta, N}} \sum_{k=0}^{N-\ell} \zeta^{k} Z_{B, k}\left(\mathcal{S}_{\ell}\right) Z_{\zeta, N-\ell-k}
$$

where we note that the $k$-particle bosonic partition function for the levels $\mathcal{S}_{\ell}$ appears in both fermionic and bosonic correlations.

Alternatively, the APFs $Z_{F, k}^{\backslash \mathcal{S}_{\ell}}, Z_{B, k}^{\backslash \mathcal{S}_{\ell}}$, and $Z_{B, k}^{\cup \mathcal{S}_{\ell}}$ can be computed recursively using Eqs. (38) and (41). This has the potential to simplify the calculation of the related full joint probability distribution, as it only requires calculating the corresponding APF with a number of particles in the range $k=0, \ldots, N$.

\section{Simplification for degenerate levels}

The expressions derived in the previous section have a very simple form when the involved levels are degenerate, i.e., if we consider correlations between the set of levels $\left\{j^{(0)}, \ldots, j^{(\ell-1)}\right\}$, where $\epsilon_{j^{(s)}}=\epsilon_{j}$ for $s \in\{0, \ldots, \ell-1\}$. The canonical partition function of $N$ bosons in $\ell$ degenerate energy levels is

$$
Z_{B, N}\left(\left\{j^{(0)}, \ldots, j^{(\ell-1)}\right\}\right)=\left(\begin{array}{c}
N+\ell-1 \\
\ell-1
\end{array}\right) e^{-\beta \epsilon_{j} N},
$$

and thus such correlations for fermions and bosons can be computed directly from Eq. (46) as

$$
\left\langle n_{j^{(0)}} \ldots n_{j^{(\ell-1)}}\right\rangle_{\zeta, N}=\frac{1}{Z_{\zeta, N}} \sum_{k=\ell}^{N} \zeta^{k-\ell}\left(\begin{array}{c}
k-1 \\
\ell-1
\end{array}\right) e^{-\beta \epsilon_{j} k} Z_{\zeta, N-k}
$$

where we have shifted the summation. The numerical complexity of calculating Eq. (48) differs from that of Eq. (37) or Eq. (40) by the number of multiplications and additions needed to calculate the extra factor $\left(\begin{array}{c}k-1 \\ \ell-1\end{array}\right)$, which can be viewed as a polynomial in $k$ of degree $\ell-1$. Thus calculating Eq. (48) requires an additional $\approx(N-\ell)(\ell-1)$ multiplications, and a similar number of additions which still scales linearly with $N$ for a moderate value of $\ell$.

\section{Expectation values of higher moments of degenerate levels}

Let us now focus on the case of bosons and revisit Eq. (35) considering a single energy level $\epsilon_{j}$ :

$$
\left\langle\left.\left(\begin{array}{c}
n_{j}-q+m_{j} \\
m_{j}
\end{array}\right)\right|_{B, N}=\frac{1}{Z_{B, N}} e^{-q \beta \epsilon_{j}} Z_{B, N-q}^{\cup\left\{j^{(1)}, \ldots, j^{\left(m_{j}\right)}\right\}} .\right.
$$

Similarly, using Eq. (6), we can write the APF $Z_{B, N-q}^{\cup\left\{j^{(1)}, \ldots, j^{\left(m_{j}\right)}\right\}}$ in terms of the system partition function and the bosonic APF $Z_{B, N-q}\left(\left\{j^{(1)}, \ldots, j^{\left(m_{j}\right)}\right\}\right)$ of $m_{j}$ degenerate levels. As a result, we obtain

$$
\left\langle\left(\begin{array}{c}
n_{j}-q+m_{j} \\
m_{j}
\end{array}\right)\right\rangle_{B, N}=\frac{1}{Z_{B, N}} \sum_{k=q}^{N}\left(\begin{array}{c}
k+m_{j}-q-1 \\
m_{j}-1
\end{array}\right) e^{-\beta \epsilon_{j} k} Z_{B, N-k},
$$

where $q \leqslant m_{j}$. Note that if we set both $m_{j}=\ell$ and $q=\ell$ then comparing with Eq. (48) we see that

$$
\left\langle\left(\begin{array}{c}
n_{j^{(s)}} \\
\ell
\end{array}\right)\right\rangle_{B, N}=\left\langle n_{j^{(0)}} \ldots n_{j^{(\ell-1)}}\right\rangle_{B, N},
$$

for $s \in\{0, \ldots, \ell-1\}$. This demonstrates that the correlations between degenerate levels can be expressed in terms of moments of the occupation number of any of the degenerate levels. This also helps to simplify the calculation of such moments; for example, we can write

$$
\left\langle n_{j}^{2}\right\rangle_{B, N}=\left\langle\left(\begin{array}{c}
n_{j} \\
2
\end{array}\right)\right\rangle_{B, N}+\left\langle\left(\begin{array}{c}
n_{j}+1 \\
2
\end{array}\right)\right\rangle_{B, N},
$$

which can be simplified using Eq. (50) as

$$
\left\langle n_{j}^{2}\right\rangle_{B, N}=\frac{1}{Z_{B, N}} \sum_{k=1}^{N}(2 k-1) e^{-\beta \epsilon_{j} k} Z_{B, N-k} .
$$

In the same fashion, we can write

$$
\left\langle n_{j}^{3}\right\rangle_{B, N}=\left\langle\left(\begin{array}{c}
n_{j} \\
3
\end{array}\right)\right\rangle_{B, N}+4\left\langle\left(\begin{array}{c}
n_{j}+1 \\
3
\end{array}\right)\right\rangle_{B, N}+\left\langle\left(\begin{array}{c}
n_{j}+2 \\
3
\end{array}\right)\right\rangle_{B, N}
$$

and thus

$$
\left\langle n_{j}^{3}\right\rangle_{B, N}=\frac{1}{Z_{B, N}} \sum_{k=1}^{N}\left(3 k^{2}-3 k+1\right) e^{-\beta \epsilon_{j} k} Z_{B, N-k} .
$$

\section{DECOMPOSITION OF LEVEL CORRELATIONS INTO OCCUPATION NUMBERS}

In the previous section, we illustrated that the joint probability distributions of the occupation numbers and corresponding level correlations can be represented by auxiliary partition functions. The APF is distinguished from the actual partition function of the $N$-particle system through either the inclusion or exclusion of a set of levels from/to the complete spectrum under study. The resulting complexity of performing an actual calculation thus depends on the size of the modified set as demonstrated by, e.g., Eq. (46).

It is known that the resulting complexity can be reduced by relating higher-order correlations between nondegenerate levels to the related level occupation numbers $[63,64]$, representing an approach similar to Wick's theorem which only holds in the grand canonical ensemble. In this section, we directly obtain many known results using the APF method and, more importantly, generalize them to deal with degenerate energy levels. 
Before we introduce the general and systematic approach to this problem, let us return to Eqs. (23) and (35) and consider two specific examples of increasing difficulty.

\section{A. Examples}

\section{Two-level correlations}

Consider the expectation value of two bosonic energy levels $j_{1}$ and $j_{2}$ where $\epsilon_{j_{1}} \neq \epsilon_{j_{2}}:\left\langle n_{j_{1}} n_{j_{2}}\right\rangle_{B, N}$. Employing Eq. (35) with $m_{j_{1}}=m_{j_{2}}=1, q_{j_{1}}=0$, and $q_{j_{2}}=1$, we find

$$
\left\langle\left(n_{j_{1}}+1\right) n_{j_{2}}\right\rangle_{B, N}=\frac{e^{-\beta \epsilon_{j_{2}}}}{Z_{B, N}} Z_{B, N-1}^{\cup\left\{j_{1}, j_{2}\right\}},
$$

and upon exchanging the values of $q_{j_{1}}$ and $q_{j_{2}}$ we have

$$
\left\langle n_{j_{1}}\left(n_{j_{2}}+1\right)\right\rangle_{B, N}=\frac{e^{-\beta \epsilon_{j_{1}}}}{Z_{B, N}} Z_{B, N-1}^{\cup\left\{j_{1}, j_{2}\right\}} .
$$

Next, eliminating the APF from the two equations yields our final result:

$$
\left\langle n_{j_{1}} n_{j_{2}}\right\rangle_{B, N}=-\frac{e^{\beta \epsilon_{j_{1}}}\left\langle n_{j_{1}}\right\rangle_{B, N}-e^{\beta \epsilon_{j_{2}}}\left\langle n_{j_{2}}\right\rangle_{B, N}}{e^{\beta \epsilon_{j_{1}}}-e^{\beta \epsilon_{j_{2}}}} .
$$

Similarly, if the levels are fermionic, we use Eq. (23) with $\left(\gamma_{j_{1}}, \gamma_{j_{2}}\right)=(0,1)$ and $(1,0)$ to find

$$
\left\langle n_{j_{1}} n_{j_{2}}\right\rangle_{F, N}=\frac{e^{\beta \epsilon_{j_{1}}}\left\langle n_{j_{1}}\right\rangle_{F, N}-e^{\beta \epsilon_{j_{2}}}\left\langle n_{j_{2}}\right\rangle_{F, N}}{e^{\beta \epsilon_{j_{1}}}-e^{\beta \epsilon_{j_{2}}}} .
$$

These known results $[63,64]$ are thus obtainable within the APF approach with a few lines of algebra by generating a set of independent equations. We now extend this idea to three energy levels.

\section{Three-level correlations}

The previous example for bosons is modified by adding a third level $j_{3}$ with $m_{j_{3}}=1$ and $\epsilon_{j_{3}}$ that is different than both $\epsilon_{j_{1}}$ and $\epsilon_{j_{2}}$. Setting $\left(q_{j_{1}}, q_{j_{2}}, q_{j_{3}}\right)=(1,0,1)$ and $(1,1,0)$ in Eq. (35) gives the two equations

$$
\begin{aligned}
\left\langle n_{j_{1}}\left(n_{j_{2}}+1\right) n_{j_{3}}\right\rangle_{B, N}=\frac{e^{-\beta\left(\epsilon_{j_{1}}+\epsilon_{j_{3}}\right)}}{Z_{B, N}} Z_{B, N-2}^{\cup\left\{j_{1}, j_{2}, j_{3}\right\}}, \\
\left\langle n_{j_{1}} n_{j_{2}}\left(n_{j_{3}}+1\right)\right\rangle_{B, N}=\frac{e^{-\beta\left(\epsilon_{j_{1}}+\epsilon_{j_{2}}\right)}}{Z_{B, N}} Z_{B, N-2}^{\cup\left\{j_{1}, j_{2}, j_{3}\right\}},
\end{aligned}
$$

respectively. Solving for $\left\langle n_{j_{1}} n_{j_{2}} n_{j_{3}}\right\rangle_{B, N}$ leads to

$$
\left\langle n_{j_{1}} n_{j_{2}} n_{j_{3}}\right\rangle_{B, N}=-\frac{e^{\beta \epsilon_{j_{2}}}\left\langle n_{j_{1}} n_{j_{2}}\right\rangle_{B, N}-e^{\beta \epsilon_{j_{3}}}\left\langle n_{j_{1}} n_{j_{3}}\right\rangle_{B, N}}{e^{\beta \epsilon_{j_{2}}}-e^{\beta \epsilon_{j_{3}}}},
$$

which can be further broken down into single-level occupation numbers by application of Eq. (56).

A slightly modified approach can be used if two of the energy levels are degenerate, $\epsilon_{j_{2}}=\epsilon_{j_{3}} \neq \epsilon_{j_{1}}$. As above, we denote degenerate levels via superscript and we relabel $j_{2}=j_{2}^{(0)}$ and $j_{3}=j_{2}^{(1)}$. Then it is clear that $\left\langle n_{j_{1}} n_{j_{2}(0)}\right\rangle_{B, N}=$ $\left\langle n_{j_{1}} n_{j_{2}^{(1)}}\right\rangle_{B, N}$ and Eq. (60) is not immediately applicable. However, this can be resolved by replacing one of the choices of $\left(q_{j_{1}}, q_{j_{2}^{(0)}}, q_{j_{2}^{(1)}}\right)$, say $(1,1,0)$, with $(0,1,1)$, which gives

$$
\left\langle n_{j_{1}} n_{j_{2}(0)} n_{j_{2}(1)}\right\rangle_{B, N}=-\frac{e^{\beta \epsilon_{j_{1}}\left\langle n_{j_{1}} n_{j_{2}}\right\rangle_{B, N}-e^{\beta \epsilon_{j_{2}}}\left\langle n_{j_{2}^{(0)}} n_{j_{2}^{(1)}}\right\rangle_{B, N}}}{e^{\beta \epsilon_{j_{1}}}-e^{\beta \epsilon_{j_{2}}}} .
$$

We now turn to the general decomposition of $\ell$-level correlations into functions of the occupation numbers of the energy levels for both the nondegenerate and degenerate spectra.

\section{B. Systematic approach}

In the following we show that Eqs. (6), (18), and (19) can be directly employed to systematically relate higher-order correlations to level occupation numbers. We begin by considering the set of levels $\mathcal{S}_{\ell}=\left\{j_{1}, j_{2}, \ldots, j_{\ell}\right\}$ and relate the corresponding $\ell$-point correlations to the $r$-point correlations for any nonempty subset of levels $\mathcal{S}_{r}=\left\{i_{1}, i_{2}, \ldots, i_{r}\right\} \subset \mathcal{S}_{\ell}$.

Imposing fermionic level statistics on the spectrum $\mathcal{S}$, we can relate the correlations $\left\langle n_{j_{1}} n_{j_{2}} \ldots n_{j_{\ell}}\right\rangle_{F, N}$ to $\left\langle n_{i_{1}} n_{i_{2}} \ldots n_{i_{r}}\right\rangle_{F, N}$ by connecting their corresponding APFs, i.e., $Z_{F, N-\ell}^{\backslash \mathcal{S}_{\ell}}$ and $Z_{F, N-r}^{\backslash \mathcal{S}_{r}}$. This can be achieved by building $Z_{F, N-1}^{\backslash \mathcal{S}_{r}}$ via Eq. (6) to combine the APFs of $\mathcal{S} \backslash \mathcal{S}_{\ell}$ with that of $\mathcal{S}_{\ell} \backslash \mathcal{S}_{r}$ through

$$
Z_{F, N-r}^{\backslash \mathcal{S}_{r}}=\sum_{k=0}^{\ell-r} Z_{F, k}^{\backslash \mathcal{S}_{r}}\left(\mathcal{S}_{\ell}\right) Z_{F, N-r-k}^{\backslash \mathcal{S}_{\ell}}
$$

We note the upper limit in the previous summation is $\ell-$ $r$, where in general it should be $k_{\max }=\min (\ell-r, N-r)$ [Eq. (6)]. This is because the fermionic APF $Z_{F, k}^{\backslash \mathcal{S}_{r}}\left(\mathcal{S}_{\ell}\right)$ cannot describe more than $\ell-r$ particles, as this is the number of levels that are in $\mathcal{S}_{\ell} \backslash \mathcal{S}_{r}$. Further, for $N<\ell$ the correlations $\left\langle n_{j_{1}} n_{j_{2}} \ldots n_{j_{\ell}}\right\rangle_{N}=0$ for general particle statistics. As a result, we only consider $\ell$-point correlations with $N \geqslant \ell$. Moreover, we can obtain $Z_{F, k}^{\backslash \mathcal{S}_{r}}\left(\mathcal{S}_{\ell}\right)$ by removing the contribution of the levels $\mathcal{S}_{r}$ from the APF of $\mathcal{S}_{\ell}$, using Eq. (18), as

$$
Z_{F, k}^{\backslash \mathcal{S}_{r}}\left(\mathcal{S}_{\ell}\right)=\sum_{m=0}^{k}(-1)^{m} Z_{B, m}\left(\mathcal{S}_{r}\right) Z_{F, k-m}\left(\mathcal{S}_{\ell}\right) .
$$

If we isolate the last term in the summation in Eq. (62), i.e., $Z_{F, \ell-r}^{\backslash \mathcal{S}_{r}}\left(\mathcal{S}_{\ell}\right) Z_{F, N-\ell}^{\backslash \mathcal{S}_{\ell}}$, while substituting for $Z_{F, k}^{\backslash \mathcal{S}_{r}}\left(\mathcal{S}_{\ell}\right)$, using Eq. (63) in all other terms, we find

$$
\begin{aligned}
Z_{F, N-r}^{\backslash \mathcal{S}_{r}}= & \sum_{k=0}^{\ell-r-1} \sum_{m=0}^{k}(-1)^{m} Z_{B, m}\left(\mathcal{S}_{r}\right) Z_{F, k-m}\left(\mathcal{S}_{\ell}\right) Z_{F, N-r-k}^{\backslash \mathcal{S}_{\ell}} \\
& +Z_{F, \ell-r}^{\backslash \mathcal{S}_{r}}\left(\mathcal{S}_{\ell}\right) Z_{F, N-\ell}^{\backslash \mathcal{S}_{\ell}} .
\end{aligned}
$$

After changing the order of the summations and shifting the indices $k \rightarrow k-r+1$ and $m \rightarrow m-r$, we obtain the unwieldy expression

$$
\begin{aligned}
Z_{F, N-r}^{\backslash \mathcal{S}_{r}}= & (-1)^{r-1} \sum_{m=r}^{\ell-1}(-1)^{m+1} Z_{B, m-r}\left(\mathcal{S}_{r}\right) \\
& \times \sum_{k=m-1}^{\ell-2} Z_{F, k-m+1}\left(\mathcal{S}_{\ell}\right) Z_{F, N-k-1}^{\backslash \mathcal{S}_{\ell}}+Z_{F, \ell-r}^{\backslash \mathcal{S}_{r}}\left(\mathcal{S}_{\ell}\right) Z_{F, N-\ell}^{\backslash \mathcal{S}_{\ell}}
\end{aligned}
$$

Next, we substitute for $Z_{F, N-\ell}^{\backslash \mathcal{S}_{\ell}}$ and $Z_{F, N-r}^{\backslash \mathcal{S}_{r}}$, using Eq. (22) and the fully occupied fermionic APF $Z_{F, \ell-r}^{\backslash \mathcal{S}_{r}}\left(\mathcal{S}_{\ell}\right)=$ $e^{-\beta \sum_{j_{v} \in \mathcal{S}_{\ell} \backslash \mathcal{S}_{r}} \epsilon_{j_{\nu}}}$. Multiplying the result by $e^{-\beta \sum_{i_{\nu} \in \mathcal{S}_{r}} \epsilon_{i_{\nu}}} / Z_{F, N}$ 
yields

$$
Y_{F, 0}\left(\mathcal{S}_{\ell}\right)+\sum_{m=r}^{\ell-1} A_{m}\left(\mathcal{S}_{r}\right) Y_{F, m}\left(\mathcal{S}_{\ell}\right)=b_{F}\left(\mathcal{S}_{r}\right)
$$

where

$$
\begin{aligned}
Y_{F, 0}\left(\mathcal{S}_{\ell}\right) & =\left\langle n_{j_{1}} n_{j_{2}} \ldots n_{j_{\ell}}\right\rangle_{F, N} \\
Y_{F, 1 \leqslant m \leqslant \ell-1}\left(\mathcal{S}_{\ell}\right) & =\frac{(-1)^{m+1}}{Z_{F, N}} \sum_{k=m-1}^{\ell-2} Z_{F, k-m+1}\left(\mathcal{S}_{\ell}\right) Z_{F, N-k-1}^{\backslash \mathcal{S}_{\ell}}
\end{aligned}
$$

are independent of $\mathcal{S}_{r}$. Therefore, for each choice of the subset $\mathcal{S}_{r}$ we can write the linear nonhomogeneous equation (66) in the $\ell$ variables $Y_{F, m}$ with coefficients $A_{0}=1, A_{0<m<r}=$ 0 , and $A_{r \leqslant m \leqslant \ell-1}\left(\mathcal{S}_{r}\right)=(-1)^{r-1} e^{-\beta \sum_{i v \in \mathcal{S}_{r}} \epsilon_{i_{v}}} Z_{B, m-r}\left(\mathcal{S}_{r}\right)$. The homogeneity of the linear equation is violated by the term $b_{F}\left(\mathcal{S}_{r}\right)=\left\langle n_{i_{1}} n_{i_{2}} \ldots n_{i_{r}}\right\rangle_{F, N}$.

With this formulation, we observe that for any of the $2^{\ell}-2$ choices of $\mathcal{S}_{r}$ we can write a linear equation in the same $\ell$ variables $Y_{F, m}$, where $Y_{F, 0}=\left\langle n_{j_{1}} n_{j_{2}} \ldots n_{j_{\ell}}\right\rangle_{F, N}$ is the $\ell$-point correlation while the remaining $\ell-1$ variables are auxiliary and depend symmetrically on the levels in $\mathcal{S}_{\ell}$. Also, the $r$ point correlation $\left\langle n_{i_{1}} n_{i_{2}} \ldots n_{i_{r}}\right\rangle_{F, N}$ of the levels in $\mathcal{S}_{r}$ plays the role of the nonhomogeneous term in the linear equation and the coefficients $A_{m}$ of the equation can be determined by the bosonic APFs of $\mathcal{S}_{r}$. An analogous expression can be obtained for bosonic statistics, with the same coefficients $A_{m}$ :

$$
Y_{B, 0}\left(\mathcal{S}_{\ell}\right)+\sum_{m=r}^{\ell-1} A_{m}\left(\mathcal{S}_{r}\right) Y_{B, m}\left(\mathcal{S}_{\ell}\right)=b_{B}\left(\mathcal{S}_{r}\right),
$$

where, in this case, the variables are

$$
\begin{aligned}
Y_{B, 1 \leqslant m \leqslant \ell-1}\left(\mathcal{S}_{\ell}\right) & = \\
& \frac{(-1)^{m+1}}{Z_{B, N}} \sum_{k=m-1}^{\ell-2}(-1)^{k} Z_{F, k-m+1}\left(\mathcal{S}_{\ell}\right) Z_{B, N-k-1}^{\cup \mathcal{S}_{\ell}},
\end{aligned}
$$

$$
\mathbb{V}\left(\alpha_{1}, \ldots, \alpha_{\ell}\right)=\left|\begin{array}{cccc}
1 & \alpha_{1} & \ldots & \alpha_{1}^{\ell-1} \\
\vdots & \vdots & \ddots & \vdots \\
1 & \alpha_{\ell} & \ldots & \alpha_{\ell}^{\ell-1}
\end{array}\right|=\prod_{i<j}\left(\alpha_{j}-\alpha_{i}\right)
$$

Thus the fermionic $\ell$-level correlation can be simplified as

$$
\left\langle n_{j_{1}} n_{j_{2}} \ldots n_{j_{\ell}}\right\rangle_{F, N}=\sum_{r=1}^{\ell} \frac{\left\langle n_{j_{r}}\right\rangle_{F, N}}{\prod_{k \neq r}\left[1-e^{\beta\left(\epsilon_{j_{k}}-\epsilon_{j_{r}}\right)}\right]} .
$$

This expression was also recently derived using an elegant second quantization scheme [63].

An equivalent procedure can be performed for bosons, again using the set $\mathcal{S}_{r=1}$ to yield $\ell$ linearly independent equations:

$$
\left\langle n_{j_{r}}\right\rangle_{B, N}=\sum_{m=0}^{\ell-1} e^{-m \beta \epsilon_{j_{r}}} Y_{B, m}\left(\mathcal{S}_{\ell}\right),
$$

with $\quad Y_{B, 0}\left(\mathcal{S}_{\ell}\right)=(-1)^{\ell-1}\left\langle n_{j_{1}} n_{j_{2}} \ldots n_{j_{\ell}}\right\rangle_{B, N} \quad$ and $\quad b_{B}\left(\mathcal{S}_{r}\right)=$ $(-1)^{r-1}\left\langle n_{i_{1}} n_{i_{2}} \ldots n_{i_{r}}\right\rangle_{B, N}$ (see Appendix B for a complete derivation).

\section{Nondegenerate levels}

Consider the set $\mathcal{S}_{\ell}$ specifying a set of distinct energy levels and choose $\mathcal{S}_{r=1}$ such that it contains only one of the $\ell$ levels in $\mathcal{S}_{\ell}$. We can use Eq. (66) or (69) to construct a set of $\ell$ linear equations each corresponding to one level $j_{s} \in \mathcal{S}_{\ell}$ with energy $\epsilon_{j_{s}}$. For fermions the equations are

$$
\left\langle n_{j_{s}}\right\rangle_{F, N}=\sum_{m=0}^{\ell-1} e^{-m \beta \epsilon_{j_{s}}} Y_{F, m}\left(\mathcal{S}_{\ell}\right),
$$

where the coefficients $A_{m}$ were obtained from the singlelevel bosonic $\operatorname{APF} Z_{B, m}\left(\left\{j_{s}\right\}\right)=e^{-m \beta \epsilon_{j_{s}}}$ in Eq. (12). Therefore, using the set of the $\ell$ independent linear equations in $\ell$ variables defined by Eq. (71), we can solve for $Y_{F, 0}\left(\mathcal{S}_{\ell}\right)=$ $\left\langle n_{j_{1}} n_{j_{2}} \ldots n_{j_{\ell}}\right\rangle_{F, N}$ as

$$
\left\langle n_{j_{1}} \ldots n_{j_{\ell}}\right\rangle_{F, N}=\frac{\left|\begin{array}{cccc}
\left\langle n_{j_{1}}\right\rangle_{F, N} & e^{-\beta \epsilon_{j_{1}}} & \ldots & e^{-\beta(\ell-1) \epsilon_{j_{1}}} \\
\vdots & \vdots & \ddots & \vdots \\
\left\langle n_{j_{\ell}}\right\rangle_{F, N} & e^{-\beta \epsilon_{j_{\ell}}} & \ldots & e^{-\beta(\ell-1) \epsilon_{j_{\ell}}}
\end{array}\right|}{\left|\begin{array}{cccc}
1 & e^{-\beta \epsilon_{j_{1}}} & \ldots & e^{-\beta(\ell-1) \epsilon_{j_{1}}} \\
\vdots & \vdots & \ddots & \vdots \\
1 & e^{-\beta \epsilon_{j_{\ell}}} & \ldots & e^{-\beta(\ell-1) \epsilon_{j_{\ell}}}
\end{array}\right|} .
$$

This result was recently obtained by Giraud, Grabsch, and Texier [64], using the properties of the Schur functions and it can be simplified using Vandermonde determinants:

$$
\begin{aligned}
& \left\langle n_{j_{1}} n_{j_{2}} \ldots n_{j_{\ell}}\right\rangle_{F, N}=\sum_{s=1}^{\ell}(-1)^{s-1}\left\langle n_{j_{s}}\right\rangle_{F, N} e^{-\beta \sum_{j_{i} \neq j_{s}} \epsilon_{j_{i}}}
\end{aligned}
$$

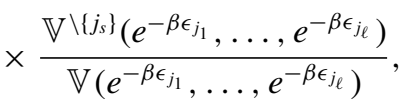

where

such that $\left\langle n_{j_{1}} n_{j_{2}} \ldots n_{j_{\ell}}\right\rangle_{B, N}=(-1)^{\ell-1} Y_{B, 0}\left(\mathcal{S}_{\ell}\right)$ can also be expressed in terms of determinants leading to [64]

$$
\left\langle n_{j_{1}} n_{j_{2}} \ldots n_{j_{\ell}}\right\rangle_{B, N}=(-1)^{\ell-1} \sum_{r=1}^{\ell} \frac{\left\langle n_{j_{r}}\right\rangle_{B, N}}{\prod_{k \neq r}\left[1-e^{\beta\left(\epsilon_{j_{k}}-\epsilon_{j_{r}}\right)}\right]} .
$$

\section{Degenerate levels}

Up until this point we have considered $\ell$-point level correlations in two opposite regimes.

(I) When all $\ell$ levels are degenerate Eq. (48) provides a direct route to the correlations through the determination of all partition functions up to $N$ particles. 
(II) When all $\ell$ levels are distinct, an associated set of $\ell$ linear equations yields the correlations in terms of individual level occupation numbers.

The independence of these linear equations, and thus the existence of a unique solution, is violated in the presence of degeneracy.

We now study the most general possible $\ell$-level correlation function defined by the set $\mathcal{S}_{\ell}$ which could include both degenerate and nondegenerate levels. Consider the subset of level indices $\mathcal{S}_{m_{i}}=\left\{i^{(0)}, \ldots, i^{\left(m_{i}-1\right)}\right\} \subset \mathcal{S}_{\ell}$ which contains $m_{i}>1$ degenerate levels (for $m_{i}=1$, we reproduce the nondegenerate analysis discussed above). As a result, the corresponding $m_{i}$ equations, out of the total set of $\ell$ linear equations defined by Eqs. (71) and (76) for fermions and bosons, respectively, are identical, as they are distinguished from each other only via the energies of the involved levels and their occupation numbers. Moreover, $\mathcal{S}_{\ell}$ could contain multiple subsets of degenerate energy levels, further complicating the problem. Equations (75) and (77) cannot be applied in this case, as is apparent from their vanishing denominators whenever $\epsilon_{j_{k}}=\epsilon_{j_{r}}$.

To resolve the complication introduced by degenerate subsets, we generalize the procedure in Sec. III A 2 to treat the case where a three-level correlation contained a subset of two degenerate levels. More explicitly, we relate the $\ell$-point correlations not only to the occupation numbers of the degenerate subsets, but to all of the $m_{i}$ distinct $r$-point correlations between the degenerate levels with $2 \leqslant r \leqslant m_{i}$. This is useful as we have already introduced Eq. (48), which simplifies the calculation of the correlations between degenerate energy levels for fermionic and bosonic statistics. In addition, it will result in the generation of $m_{i}$ new independent equations that could be used to calculate $\ell$-point correlations.

Accordingly, for any choice of $\mathcal{S}_{r}=\left\{i^{(0)}, \ldots, i^{(r-1)}\right\} \subset$ $\mathcal{S}_{m_{i}} \subset \mathcal{S}_{\ell}$, the corresponding coefficients in the constructed linear equations are $A_{0}=1, A_{0<m<r}=0$ and

$$
A_{r \leqslant m \leqslant \ell-1}\left(\mathcal{S}_{r}\right)=(-1)^{r-1}\left(\begin{array}{c}
m-1 \\
r-1
\end{array}\right) e^{-m \beta \epsilon_{i}},
$$

where $\epsilon_{i}$ is the energy of all degenerate levels in $\mathcal{S}_{m_{i}}$ and we have substituted for $Z_{B, m-r}\left(\left\{i^{(0)}, \ldots, i^{(r)}\right\}\right)=\left(\begin{array}{c}m-1 \\ r-1\end{array}\right) e^{-(m-r) \beta \epsilon_{i}}$ a bosonic partition function of $r$ degenerate levels.

The nonhomogeneities $b_{\zeta}\left(\mathcal{S}_{r}\right)=$ $(-\zeta)^{r-1}\left\langle n_{i^{(1)}} n_{i^{(2)}} \ldots n_{i^{(r-1)}}\right\rangle_{\zeta, N}$ can be calculated using Eq. (48), and thus the original set of $m_{i}$ identical equations can now be replaced with the following $m_{i}$ independent equations:

$$
Y_{\zeta, 0}\left(\mathcal{S}_{\ell}\right)+\sum_{m=r}^{\ell-1}(-1)^{r-1}\left(\begin{array}{c}
m-1 \\
r-1
\end{array}\right) e^{-m \beta \epsilon_{i}} Y_{\zeta, m}\left(\mathcal{S}_{\ell}\right)=b_{\zeta}\left(\mathcal{S}_{r}\right),
$$

for fermions $(\zeta=-1)$ and bosons $(\zeta=+1)$.

To illustrate how this works in practice, consider the fourpoint correlation of the levels $\left\{j_{1^{(0)}}, j_{1^{(1)}}, j_{1^{(2)}}, j_{2}\right\}$, labeling distinct energies $\epsilon_{1}$ and $\epsilon_{2}$. The resulting new set of $\ell$ equations can be solved for both fermions and bosons to give

$$
\left\langle n_{j_{1}(0)} n_{j_{1}(1)} n_{j_{1}(2)} n_{j_{2}}\right\rangle_{\zeta, N}=(-\zeta) \frac{\left|\begin{array}{cccc}
\left\langle n_{j_{1}(0)} n_{j_{1}(1)} n_{j_{1}(2)}\right\rangle_{\zeta, N} & 0 & 0 & e^{-3 \beta \epsilon_{j_{1}}} \\
(-\zeta)\left\langle n_{j_{1}(0)} n_{j_{1}(1)}\right\rangle_{\zeta, N} & 0 & -e^{-2 \beta \epsilon_{j_{1}}} & -2 e^{-3 \beta \epsilon_{j_{1}}} \\
\left\langle n_{j_{1}(0)}\right\rangle_{\zeta, N} & e^{-\beta \epsilon_{j_{1}}} & e^{-2 \beta \epsilon_{j_{1}}} & e^{-3 \beta \epsilon_{j_{1}}} \\
\left\langle n_{j_{2}}\right\rangle_{\zeta, N} & e^{-\beta \epsilon_{j_{2}}} & e^{-2 \beta \epsilon_{j_{2}}} & e^{-3 \beta \epsilon_{j_{2}}}
\end{array}\right|}{\left|\begin{array}{cccc}
1 & 0 & 0 & e^{-3 \beta \epsilon_{j_{1}}} \\
1 & 0 & -e^{-2 \beta \epsilon_{j_{1}}} & -2 e^{-3 \beta \epsilon_{j_{1}}} \\
1 & e^{-\beta \epsilon_{j_{1}}} & e^{-2 \beta \epsilon_{j_{1}}} & e^{-3 \beta \epsilon_{j_{1}}} \\
1 & e^{-\beta \epsilon_{j_{2}}} & e^{-2 \beta \epsilon_{j_{2}}} & e^{-3 \beta \epsilon_{j_{2}}}
\end{array}\right|} .
$$

\section{APPLICATIONS}

To illustrate the applicability of our results for degenerate noninteracting systems of particles with fixed number and highlight the practical usage of Eq. (80) we consider a onedimensional tight-binding chain of $N$ spinful bosons hopping over $L$ lattice sites. Code, scripts, and data used to produce all figures for the bosonic chain results can be found online [84]. The bosonic chain is subject to a static external magnetic field $B$ applied along the $z$ axis. Spin- $S$ bosons are described by the Hamiltonian

$$
\widehat{H}=-t \sum_{\alpha, \sigma}\left(\hat{a}_{\alpha+1, \sigma}^{\dagger} \hat{a}_{\alpha, \sigma}+\text { H.c. }\right)-h \sum_{\alpha, \sigma, \sigma^{\prime}} \hat{a}_{\alpha, \sigma}^{\dagger} S_{\sigma, \sigma^{\prime}}^{z} \hat{a}_{\alpha, \sigma^{\prime}},
$$

where $\hat{a}_{\alpha, \sigma}^{\dagger}$ and $\hat{a}_{\alpha, \sigma}$ are creation and annihilation operators for a boson at site $\alpha$ with $\sigma \in\{-S, \ldots, 0, \ldots, S\}$ satisfying $\left[a_{\alpha, \sigma}, a_{\alpha^{\prime}, \sigma^{\prime}}^{\dagger}\right]=\delta_{\alpha, \alpha^{\prime}} \delta_{\sigma, \sigma^{\prime}}$ and $t$ measures the hopping amplitude. $S_{\sigma, \sigma^{\prime}}^{z}=\sigma \delta_{\sigma, \sigma^{\prime}}$ are the matrix elements of the diagonal $z$ projection of the spin-S representation of the spin operator $\hat{\boldsymbol{S}}$.
Here, $h=g \mu_{\mathrm{B}} B$, where $g$ is the corresponding spin- $S g$ factor and $\mu_{B}$ is the Bohr magneton. We employ periodic boundary conditions, such that $\hat{a}_{L+1, \sigma}=\hat{a}_{1, \sigma}$, and to avoid having an unbalanced nondegenerate excited state we fix the parity of $L$ to be odd.

The tight-binding Hamiltonian in Eq. (81) can be diagonalized:

$$
\widehat{H}=\sum_{j, \sigma} \epsilon_{j, \sigma} n_{j, \sigma},
$$

where $\hat{n}_{j, \sigma}$ counts the number of bosons with energy

$$
\epsilon_{j, \sigma}=-2 t \cos \left(\frac{2 \pi j}{L}\right)-h \sigma,
$$

and $j$ runs over the finite set $\mathcal{S}=\left\{-\frac{L-1}{2}, \ldots, 0, \ldots, \frac{L-1}{2}\right\}$ when $L$ is odd such that $|\mathcal{S}|=L$. An examination of the single-particle spectrum shows that each energy level, except the ground state $\epsilon_{0, \sigma}=-2 t-h \sigma$, is twofold degenerate, where $\epsilon_{-j, \sigma}=\epsilon_{j, \sigma}$, a result of the right-left symmetry of the 
chain. Turning off the magnetic field and fixing $S>0$ gives rise to an extra degeneracy factor of $(2 S+1)$ that affects all levels.

For all numerical results presented in this section, we fix $L=1001$ and $N=1000$ and measure the inverse temperature $\beta=\frac{1}{k_{\mathrm{B}} T}$ in units of $1 / t$, where $T$ is the absolute temperature and $k_{\mathrm{B}}$ is the Boltzmann constant.

\section{A. Spinless bosons $(S=0)$}

We begin with the study of spinless bosons, where the model is insensitive to the applied magnetic field and we can drop the subscript $\sigma$ without loss of generality. Using the single-particle spectrum defined in Eq. (83) with $\sigma=0$ and $h=0$ in combination with Eq. (27), we calculate the joint probability distribution $\mathcal{P}_{B, n_{0}, n_{1}}$ of the occupation numbers of the ground state and the first excited state, where we choose the level $j=1$ out of the two degenerate levels $j= \pm 1$. Note that we do not bother to use the superscript notation to distinguish degenerate level indices $\left(1 \equiv 1^{(0)},-1 \equiv 1^{(1)}\right)$ here as there are no ambiguities due to the sign of the index $j$.

The calculation proceeds by obtaining the APFs $Z_{B, k}^{\backslash\{0,1\}}$ for $0 \leqslant k \leqslant N$ using the recursion relation Eq. (41), where the factors $C_{k}$ are calculated using the spectrum $\mathcal{S} \backslash\{0,1\}$. The resulting distribution is

$$
\mathcal{P}_{B, n_{0}, n_{1}}=\frac{e^{-\beta\left(\epsilon_{0} n_{0}+\epsilon_{1} n_{1}\right)}}{Z_{B, N}} Z_{B, N-n_{0}-n_{1}}^{\backslash\{0,1\}},
$$

where $Z_{B, N}$ can be found by enforcing normalization.

We expect Eq. (84) to exhibit interesting features at low temperature where the particles are mostly occupying the ground state with some fluctuations amongst the low-lying energy levels. To obtain an estimate of low in this context, we choose a value of the inverse temperature $\beta$ such that the ground state has a macroscopic occupation corresponding to $50 \%$ of the particles. We compare the ratio of the Boltzmann factors of having all particles in the ground state with that of having $N / 2$ particles in the first excited state and the rest in the ground state. Setting the ratio of these factors $e^{-\beta\left(\epsilon_{0}-\epsilon_{1}\right) N / 2}$ to $\approx 0.1$ suggests $\beta \sim 100 / t$. The results are illustrated in the left panel of Fig. 1, where the relative broadness of the distribution can be attributed to the degeneracy of the first exited level $j= \pm 1$.

If we now calculate the three-level joint probability distribution $\mathcal{P}_{B, n_{0}, n_{1}, n_{-1}}$ and consider the fixed slice with $n_{-1}=0$, as presented in the right panel of Fig. 1, we see that the distribution becomes significantly sharper, as blocking the level $j=-1$ makes the resulting non-normalized conditional distribution more sensitive to the conservation of the total number of particles.

We now turn to the calculation of the two-level connected correlation function for our bosonic system:

$$
\mathcal{C}\left(n_{i}, n_{j}\right)=\left\langle n_{i} n_{j}\right\rangle_{B, N}-\left\langle n_{i}\right\rangle_{B, N}\left\langle n_{j}\right\rangle_{B, N}
$$

The first step is to obtain the system partition function $Z_{B, k}$, recursively, using Eq. (41) starting from $Z_{B, 0}$ up to $Z_{B, N}$. The occupation numbers $\left\langle n_{j}\right\rangle_{B, N}$ can then be easily calculated using Eq. (40). All that remains is to calculate the two-point correlations using Eq. (56) for the nondegenerate levels. For $n_{1}$

$0 \quad 200 \quad 400 \quad 600 \quad 8001000 \quad 0 \quad 200 \quad 400 \quad 600 \quad 800 \quad 1000$

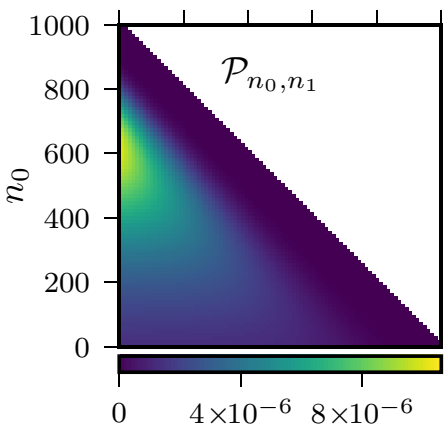

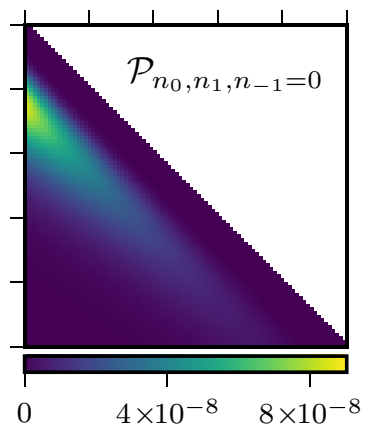

FIG. 1. The joint level probability distribution for $N=1000$ spinless bosons on a chain of $L=1001$ sites described by Eq. (82) with $\sigma=0$ and $h=0$ at inverse temperature $\beta=100 / t$. Left panel: $\mathcal{P}_{B, n_{0}, n_{1}}$. Right panel: $\mathcal{P}_{B, n_{0}, n_{1}, n_{-1}}$ projected into the plane $n_{-1}=0$.

the correlations between degenerate levels $\left(\left\langle n_{-j} n_{j}\right\rangle_{B, N}\right)$, we use Eq. (48), with $\zeta=+1$.

The results for $\mathcal{C}\left(n_{i}, n_{j}\right)$ for all levels $i$ and $j$ at inverse temperature $\beta=1 / t$ are shown as a heat map in the lower panel of Fig. 2. Here, we chose temperature $\beta=1 / t$ in order to distribute the correlations amongst higher-energy levels. The upper panel of Fig. 2 shows $\mathcal{C}\left(n_{i}, n_{j}\right)$ as a function of $n_{i}$ for fixed $0 \leqslant j \leqslant 500$ corresponding to horizontal cuts through the lower panel. The red open circles are correlations $\mathcal{C}\left(n_{-j}, n_{j}\right)$ for the degenerate levels obtained from Eq. (48) demonstrating consistency with the rest of the graph. In the positive quadrant of the correlation heat map, we use the values of $\left\langle\left(\begin{array}{c}n_{j} \\ 2\end{array}\right)\right\rangle_{B, N}$ (marked with black circles) instead of $\left\langle n_{j}^{2}\right\rangle_{B, N}$, where the former is also consistent with surrounding data, as expected from Eq. (51), where $\left\langle n_{-j} n_{j}\right\rangle_{B, N}=\left\langle\left(\begin{array}{c}n_{j} \\ 2\end{array}\right)\right\rangle_{B, N}$ and the symmetry $\mathcal{C}\left(n_{i}, n_{j}\right)=\mathcal{C}\left(n_{-i}, n_{j}\right)$ due to the degeneracy.

\section{B. Spin-1 bosons $(S=1)$}

To illustrate the utility of auxiliary partitions functions in studying correlations in a highly degenerate spectrum, we consider the case of spin-1 bosons. In the absence of a magnetic field $(h=0)$, each level picks up a degeneracy factor of $2 S+1=3$, such that the ground state is threefold degenerate and all of the excitation levels are sixfold degenerate.

Degeneracy effects are apparent in the two-level connected correlations $\mathcal{C}\left(n_{i, \sigma}, n_{j, \sigma^{\prime}}\right)$ at $\beta=1 / t$, which we calculate for various values of $h$ as shown in Fig. 3. The top-left panel of the figure presents $\mathcal{C}\left(n_{i, \sigma}, n_{j, \sigma^{\prime}}\right)$ for $h=0$, where the choice of $\sigma$ and $\sigma^{\prime}$ matters only in the presence of a magnetic field. A comparison with the heat map of Fig. 2 shows an overall broadening and a reduction of one order of magnitude in the maximum of $\mathcal{C}\left(n_{i, \sigma}, n_{j, \sigma^{\prime}}\right)$ for $S=1$, as compared to the $S=0$ case.

Removing the energy-spin degeneracy by applying a strong magnetic field of $h=5 t$ results in a splitting of the spectrum into three bands, each with bandwidth $4 t$ and separated from each other via an energy band gap of $t$. In this case, we first focus on correlations between the levels in the lower-energy band ( $\sigma=1$, bottom-left panel of Fig. 3), and see a partial 


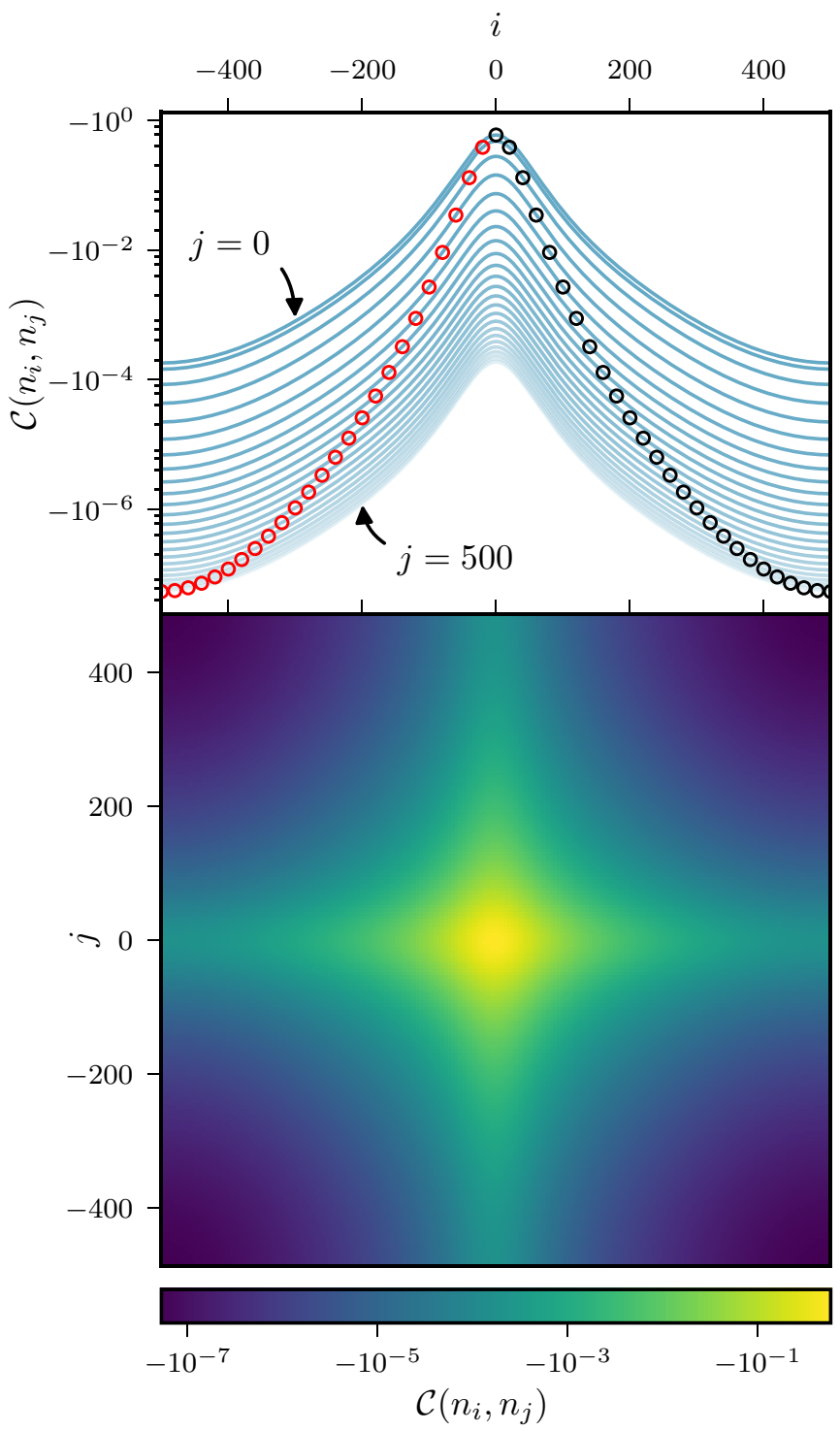

FIG. 2. Lower panel: Heat map depicting two-point connected correlations $\mathcal{C}\left(n_{i}, n_{j}\right)=\left\langle n_{i} n_{j}\right\rangle_{B, N}-\left\langle n_{i}\right\rangle_{B, N}\left\langle n_{j}\right\rangle_{B, N}$ in a system of $N=1000$ spinless bosons on a lattice of $L=1001$ sites at $\beta=1 / t$. Upper panel: Horizontal cuts from the upper half of $\mathcal{C}\left(n_{i}, n_{j}\right)$ at different values of the index $j>0$. The circled data points are calculated using Eq. (48), where the red-circled points are $\mathcal{C}\left(n_{-j}, n_{j}\right)$ for the degenerate levels $j$ and $-j$, while the black-circled points are $\lim _{i \rightarrow j} \mathcal{C}\left(n_{i}, n_{j}\right)=\left\langle\left(\begin{array}{c}n_{j} \\ 2\end{array}\right)\right\rangle_{B, N}-\left\langle n_{j}\right\rangle_{B, N}^{2}$.

recovery of the spinless bosons case (Fig. 2). Correlations that involve higher-energy levels $(\sigma=0$ and -1$)$ are orders of magnitude weaker, at the considered temperatures, as shown in the right panels of Fig. 3.

Finally, we turn to correlations between a set of levels that is partially degenerate, an interesting feature of spin-1 bosons. We consider the four-level (disconnected) correlations $\left\langle n_{i, 1} n_{j, 1} n_{j, 0} n_{j,-1}\right\rangle_{B, N}$ between the levels $\epsilon_{i, 1}, \epsilon_{j, 1}, \epsilon_{j, 0}$ and $\epsilon_{j,-1}$, where, in the absence of a magnetic field, the last three levels are degenerate for any $j$. We employ the bosonic version of Eq. (80) with $\zeta=1$ with results shown in Fig. 4. According to Eq. (35), the results that we obtain, in this case,

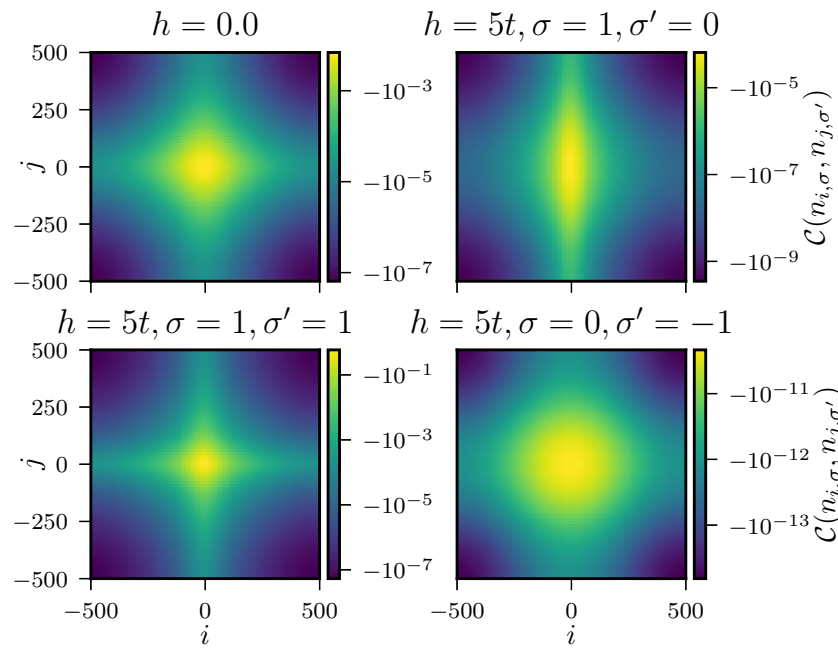

FIG. 3. Two-level connected correlation function $\mathcal{C}\left(n_{i, \sigma}, n_{j, \sigma^{\prime}}\right)$ at $\beta=1 / t$ for $N=1000$ spin- 1 bosons on $L=1001$ sites. Panels correspond to different values of $\sigma, \sigma^{\prime}$, and the applied magnetic field $\left(h=g \mu_{\mathrm{B}} B\right)$ as indicated.

also represent $\left\langle n_{i, \sigma}\left(\begin{array}{c}n_{j, \sigma^{\prime}} \\ 3\end{array}\right)\right\rangle_{B, N}$, for any $\sigma$ and $\sigma^{\prime} \in\{1,0,-1\}$. For the fully degenerate case $i=-j$, we use Eq. (48). Once more, Eq. (35) guarantees that $\left\langle n_{i, 1} n_{-i, 1} n_{-i, 0} n_{-i,-1}\right\rangle_{B, N}=$ $\left\langle\left(\begin{array}{c}n_{i, \sigma} \\ 4\end{array}\right)\right\rangle_{B, N}$. Therefore, we use $\left\langle\left(\begin{array}{c}n_{i, \sigma} \\ 4\end{array}\right)\right\rangle_{B, N}$ instead of $\left\langle n_{i, \sigma}^{4}\right\rangle_{B, N}$, for the diagonal elements of the four-level disconnected correlations presented in the lower panel of Fig. 4. The consistency of our calculations using different equations and methods described herein is demonstrated in the upper panel in analogy with Fig. 2.

\section{DISCUSSION}

In summary, we have presented a statistical theory of noninteracting identical quantum particles in the canonical ensemble, providing a unified framework that symmetrically captures both fermionic and bosonic statistics. Table I includes a listing of our most important results for fermions and bosons. We achieve this by (1) representing correlations [Eqs. (23) and (35)] and joint probability distributions [(21) and (27)] via auxiliary partition functions and (2) deriving general relations between the canonical partition function of a given spectrum and that of the auxiliary partition function describing a spectral subset, as captured by Eqs. (8), (18), and (19).

These key equations can be manipulated to simplify the derivation of the known recursive relations for partition functions in the canonical ensemble and lead immediately to generalizations and, more importantly, provide useful formulas for calculating the correlations between degenerate energy levels and for calculating higher moments of the occupation number distribution. Also, Eqs. (23) and (35) can be used to reduce the complexity order of the desired correlations, or to relate them to the occupation numbers of the involved levels and correlations between entirely degenerate levels [see Eq. (48)]. Moreover, the ability to manipulate the way an auxiliary partition function is built out of other ones allows us to construct a systematic approach towards the decomposition 


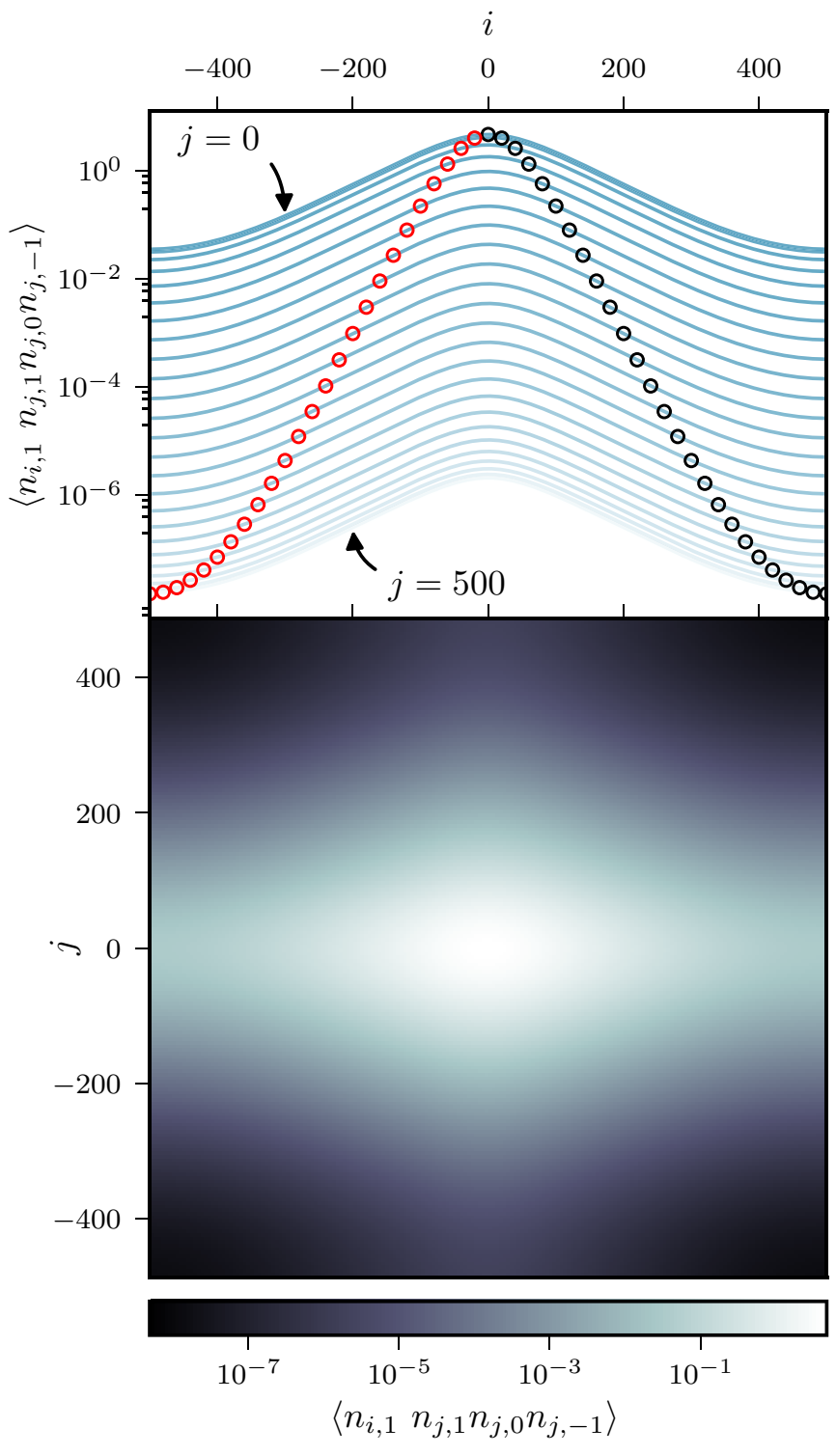

FIG. 4. Lower panel: Four-level correlations $\left\langle n_{i, 1} n_{j, 1} n_{j, 0} n_{j,-1}\right\rangle_{B, N}$ at $\beta=1 / t$ for $N=1000$ spin-1 bosons on $L=1001$ sites with no magnetic field applied $(h=0)$. Upper panel: Horizontal cuts from the upper half of the $\left\langle n_{i, 1} n_{j, 1} n_{j, 0} n_{j,-1}\right\rangle_{B, N}$ heat map at different values of the index $j>0$. The circled data points are calculated using Eq. (48), where the red-circled points are the fully degenerate case $i=-j$, while the black-circled points are $\lim _{i \rightarrow j}\left\langle n_{i, 1} n_{j, 1} n_{j, 0} n_{j,-1}\right\rangle_{B, N}=\left\langle\left(\begin{array}{c}n_{j} \\ 4\end{array}\right)\right\rangle_{B, N}$.

of many-energy-level correlations in terms of individual level occupancies. This reflects the additional constraints between energy levels due to fixed $N$ even in the absence of interactions that are not present in a grand canonical description. Thus, we present an approach to working in the canonical ensemble that includes a generalization of Wick's theorem, where we obtain previous results for nondegenerate levels $[63,64]$ and extend them to the case of a degenerate spectrum.

Interestingly, despite the substantial difference between fermionic and bosonic statistics, the resulting formulas show evident similarity. If we compare Eqs. (36), (37), and (38)
TABLE I. A summary of the main results presented in this paper that can be utilized to determine energy-level occupation numbers, correlations, and probabilities for $N$ noninteracting fermions $(F)$ and bosons $(B)$ with energy spectra $\epsilon_{i}$ with $i \in \mathcal{S}=\{1,2, \ldots, M\}$ in the canonical ensemble. Here $n_{j}$ is the occupation of the $j$ th level, $\gamma_{j_{r}}=0$ and 1 , and $0 \leqslant q_{j_{r}} \leqslant m_{j_{r}} \in \mathbb{Z}$. All computations rely on the introduction of auxiliary partition functions that describe a modified spectra or subset of levels connected to $\mathcal{S}$ through the removal of levels or the addition of degeneracy.

Fermionic results

Level correlations, Eq. (23):

$$
\begin{array}{r}
\left\langle\prod_{r=1}^{\ell}\left[n_{j_{r}} \gamma_{j_{r}}+\left(1-n_{j_{r}}\right)\left(1-\gamma_{j_{r}}\right)\right]\right\rangle_{F, N} \\
=\frac{1}{Z_{F, N}} e^{-\beta \sum_{r=1}^{\ell} \epsilon_{j_{r}} \gamma_{j_{r}}} Z_{F, N-\sum_{r=1}^{\ell} \gamma_{j_{r}}}^{\backslash\left\{j_{1}, j_{2}, \ldots, j_{\ell}\right\}} .
\end{array}
$$

Joint probability distribution, Eq. (21):

$\mathcal{P}_{F, n_{j_{1}}, n_{j_{2}}, \ldots, n_{j_{\ell}}}=\frac{e^{-\beta \sum_{r=1}^{\ell} \epsilon_{j_{r}} n_{j_{r}}}}{Z_{F, N}} Z_{F, N-\sum_{r=1}^{\ell} n_{j_{r}}}^{\backslash\left\{j_{1}, j_{2}, \ldots, j_{\ell}\right\}}$.

Auxiliary partition function, Eq. (18):

$Z_{F, N}^{\left\lfloor j_{1}, j_{2}, \ldots, j_{\ell}\right\}}=\sum_{k=0}^{N}(-1)^{k} Z_{B, k}\left(\left\{j_{1}, j_{2}, \ldots, j_{\ell}\right\}\right) Z_{F, N-k}$.

Bosonic results

Level correlations, Eq. (35):

$$
\begin{aligned}
& \left\langle\prod_{r=1}^{\ell}\left(\begin{array}{c}
n_{j_{r}}-q_{j_{r}}+m_{j_{r}} \\
m_{j_{r}}
\end{array}\right)\right\rangle_{B, N} \\
& \quad=\frac{1}{Z_{B, N}} e^{-\beta \sum_{r=1}^{\ell} \epsilon_{j_{r}} q_{j_{r}}} Z_{B, N-\sum_{r=1}^{\ell} q_{j r}}^{\left.\cup(1), \ldots, j_{1}^{\left(m_{j_{1}}\right)}, \ldots j_{\ell}^{(1)}, \ldots, j_{\ell} m_{j_{\ell}}{ }^{\left(m_{j}\right.}\right\}} .
\end{aligned}
$$

Joint probability distribution, Eq. (27):

$\mathcal{P}_{B, n_{j_{1}}, n_{j_{2}}, \ldots, n_{j_{\ell}}}=\frac{e^{-\beta \sum_{r=1}^{\ell} \epsilon_{j_{r}} n_{j_{r}}}}{Z_{B, N}} Z_{B, N-\sum_{r=1}^{\ell} n_{j r}}^{\backslash\left\{j_{1}, j_{2}, \ldots, j_{\ell}\right\}}$

Auxiliary partition function, Eq. (19):

$Z_{B, N}^{\left\lfloor j_{1}, j_{2}, \ldots, j_{\ell}\right\}}=\sum_{k=0}^{N}(-1)^{k} Z_{F, k}\left(\left\{j_{1}, j_{2}, \ldots, j_{\ell}\right\}\right) Z_{B, N-k}$.

with Eqs. (39), (40), and (41), respectively, we see that the differences between the fermionic and the bosonic formulas can be captured by simple \pm 1 factors. In view of the current theory, such similarity is associated with the interplay between fermionic and bosonic auxiliary partition functions. The inverted symmetry between the two distinct statistics is apparent via a comparison of Eqs. (13) and (14) with Eqs. (15) and (16) reflective of the fact that adding a fermionic energy level to the partition function is similar to excluding a bosonic one and vice versa.

While the focus of this paper is on quantum canonical statistics, it is worth mentioning that one can also obtain the well-known formulas of the grand canonical partition functions for both fermionic and bosonic statistics from the theory of auxiliary partition functions. For a given 
chemical potential $\mu$, applying the factor $e^{\beta \mu N}$ to Eq. (13), followed by performing the summation $\sum_{N=0}^{\infty}$, we obtain the simple recursive relation $\mathcal{Z}_{F}=\mathcal{Z}_{F}^{\backslash\{j\}}\left(1+e^{\beta\left(\mu-\epsilon_{j}\right)}\right)$, where $\mathcal{Z}_{F}=\sum_{N=0}^{\infty} e^{\beta \mu N} Z_{F, N}$ is the grand canonical partition function and $\mathcal{Z}_{F}^{\backslash\{j\}}$ is an auxiliary one. This leads immediately to $\mathcal{Z}_{F}=\prod_{j}\left(1+e^{\beta\left(\mu-\epsilon_{j}\right)}\right)$. Starting from Eq. (16), the bosonic grand canonical partition function can be obtained in a similar fashion.

The presented formulas for combining and resolving auxiliary partition functions allows for their construction via different routes which we have utilized to obtain exact expressions for the decomposition of correlations in terms of single-level occupation numbers. These different forms may also have value in overcoming the known numerical instabilities of the recursive formula for the fermionic partition function due to the influence of alternating signs [63,85]. In addition, the simplicity of the presented theory suggests a possible generalization to cover different energy-level occupation constraints beyond the fermionic and bosonic ones. This includes classical Maxwell-Boltzmann statistics in the canonical ensemble.

We envision that the results presented herein could have applications in the computation of entanglement entropy in the presence of superselection rules, as well as in modeling cold atom experiments. In the context of quantum information, the spectrum of the reduced density matrix corresponding to a mode bipartition of a state of conserved number $N$ of itinerant particles on a lattice can be associated with that of a fictional entanglement Hamiltonian. For noninteracting particles, the entanglement entropy can be obtained via the so-called correlation matrix method [86-89] which requires the evaluation of the canonical partition function of the resulting noninteracting entanglement Hamiltonian. For trapped ultracold atoms at low densities where $N$ is fixed and interactions can be neglected, the analysis of experimental results in the physically correct canonical ensemble provides improved thermometry, especially for the case of fermions.

Finally, the ability to directly study level statistics in the canonical ensemble for bosons and fermions may have pedagogical value in the teaching of statistical mechanics, where the more physical concept of a fixed number of particles is quickly jettisoned and replaced with a grand canonical reservoir for the sake of simplifying derivations.

\section{ACKNOWLEDGMENTS}

We thank D. Clougerty for bringing our attention to Ref. [69] and K. Schönhammer for discussions at an early stage of this work. We would like to thank I. Hamammu for pointing out the relation to nuclear physics. This research was supported in part by NSF Grants No. DMR-1553991 (A.D.) and No. DMR-1828489 (H.B.). All computations were performed on the Vermont Advanced Computing Core supported in part by NSF Grant No. OAC-1827314.

\section{APPENDIX A: ADDITIONAL PROOF OF EQ. (17)}

Here we provide another proof for Eq. (17) of the main text via mathematical induction. First, we substitute for $\epsilon_{j}=\epsilon_{j}^{\prime} \mp i \pi / \beta$ in Eq. (15):

$$
Z_{F, N}^{\backslash\{j\}}=\sum_{k=0}^{N} Z_{B, k}^{\prime}(\{j\}) Z_{F, N-k},
$$

where $Z_{B, k}^{\prime}(\{j\})=e^{-\beta \epsilon_{j}^{\prime} k}$ is the single-level bosonic APF. This validates Eq. (17) for the removal of a single level, i.e., for $\ell=$ 1. Next, we use Eq. (15) to remove an additional energy level, $\epsilon_{j_{\ell+1}}$, from $Z_{F, N}^{\backslash\left\{j_{1}, j_{2}, \ldots, j_{\ell}\right\}}$ and substitute for $\epsilon_{j_{\ell+1}}=\epsilon_{j_{\ell+1}}^{\prime} \mp i \pi / \beta$ :

$$
Z_{F, N}^{\backslash \mathcal{S}_{\ell+1}}=\sum_{m=0}^{N} e^{-m \beta \epsilon_{j_{\ell+1}}^{\prime}} Z_{F, N-m}^{\backslash \mathcal{S}_{\ell}},
$$

where $\mathcal{S}_{\ell}=\left\{j_{1}, j_{2}, \ldots, j_{\ell}\right\}$ and $\mathcal{S}_{\ell+1}=\left\{j_{1}, j_{2}, \ldots, j_{\ell+1}\right\}$. Now we assume that Eq. (17) holds for removing $\ell$ levels and use it to substitute for $Z_{F, N-m}^{\backslash \mathcal{S}_{\ell}}$ in the above equation. This gives

$$
Z_{F, N}^{\backslash \mathcal{S}_{\ell+1}}=\sum_{m=0}^{N} e^{-m \beta \epsilon_{j_{\ell+1}}^{\prime}} \sum_{k=0}^{N-m} Z_{B, k}^{\prime}\left(\mathcal{S}_{\ell}\right) Z_{F, N-m-k} .
$$

Performing the index change $k \rightarrow k-m$ and reordering the summations yields

$$
Z_{F, N}^{\backslash \mathcal{S}_{\ell+1}}=\sum_{k=0}^{N} \sum_{m=0}^{k} e^{-m \beta \epsilon_{j_{\ell+1}}^{\prime}} Z_{B, k-m}^{\prime}\left(\mathcal{S}_{\ell}\right) Z_{F, N-k} .
$$

From Eq. (14), the inner summation is simply $Z_{B, k}^{\prime}\left(\mathcal{S}_{\ell+1}\right)$ and thus Eq. (17) holds for removing $\ell+1$ levels, i.e.,

$$
Z_{F, N}^{\backslash \mathcal{S}_{\ell+1}}=\sum_{k=0}^{N} Z_{B, k}^{\prime}\left(\mathcal{S}_{\ell+1}\right) Z_{F, N-k} .
$$

This proves Eq. (17) in general.

\section{APPENDIX B: DERIVATION OF EQ. (69)}

Starting with the sets of levels $\mathcal{S}_{r}=\left\{i_{1}, i_{2}, \ldots, i_{r}\right\} \subset \mathcal{S}_{\ell}=$ $\left\{j_{1}, j_{2}, \ldots, j_{\ell}\right\}$ and using Eq. (19) we have

$$
Z_{B, N-r}^{\cup \mathcal{S}_{r}}=\sum_{k=0}^{\ell-r}(-1)^{k} Z_{F, k}^{\backslash \mathcal{S}_{r}}\left(\mathcal{S}_{\ell}\right) Z_{B, N-r-k}^{\cup \mathcal{S}_{\ell}} .
$$

Next, we substitute for $Z_{F, k}^{\backslash \mathcal{S}_{r}}\left(\mathcal{S}_{\ell}\right)$, using Eq. (63), except for the last term $(-1)^{\ell-r} Z_{F, \ell-r}^{\backslash \mathcal{S}_{r}}\left(\mathcal{S}_{\ell}\right) Z_{B, N-\ell}^{\cup \mathcal{S}_{\ell}}$ which we separate from the rest of the previous summation; thus we obtain

$$
\begin{aligned}
Z_{B, N-r}^{\cup \mathcal{S}_{r}}= & \sum_{k=0}^{\ell-r-1} \sum_{m=0}^{k}(-1)^{m+k} Z_{B, m}\left(\mathcal{S}_{r}\right) Z_{F, k-m}\left(\mathcal{S}_{\ell}\right) Z_{B, N-r-k}^{\cup \mathcal{S}_{\ell}} \\
& +(-1)^{\ell-r} Z_{F, \ell-r}^{\backslash \mathcal{S}_{r}}\left(\mathcal{S}_{\ell}\right) Z_{B, N-\ell}^{\cup \mathcal{S}_{\ell}} .
\end{aligned}
$$

If we rearrange the summations and perform the index change $k \rightarrow k-r+1$ and $m \rightarrow m-r$, we get

$$
\begin{aligned}
Z_{B, N-r}^{\cup \mathcal{S}_{r}}= & \sum_{m=r}^{\ell-1}(-1)^{m+1} Z_{B, m-r}\left(\mathcal{S}_{r}\right) \\
& \times \sum_{k=m-1}^{\ell-2}(-1)^{k} Z_{F, k-m+1}\left(\mathcal{S}_{\ell}\right) Z_{B, N-k-1}^{\cup \mathcal{S}_{\ell}} \\
& +(-1)^{\ell-r} Z_{F, \ell-r}^{\backslash \mathcal{S}_{r}}\left(\mathcal{S}_{\ell}\right) Z_{B, N-\ell}^{\cup \mathcal{S}_{\ell} .}
\end{aligned}
$$


Now, using Eq. (34), we substitute for $Z_{B, N-\ell}^{\cup \mathcal{S}_{\ell}}$ and $Z_{B, N-r}^{\cup \mathcal{S}_{r}}$ as well as the APF $Z_{F, \ell-r}^{\backslash \mathcal{S}_{r}}\left(\mathcal{S}_{\ell}\right)=e^{-\beta \sum_{j v} \in \mathcal{S}_{\ell} \backslash \mathcal{S}_{r}} \epsilon_{j_{v}}$. After multiplying the resulting equation by $\frac{(-1)^{r-1} e^{-\beta \sum_{i_{v} \in \mathcal{S}_{r}} \epsilon_{i_{v}}}}{Z_{B, N}}$, we can write

$$
Y_{B, 0}+\sum_{m=r}^{\ell-1} A_{B, m}\left(\mathcal{S}_{r}\right) Y_{B, m}=b_{B}\left(\mathcal{S}_{r}\right)
$$

[1] C. Kittel, Thermal Physics, 2nd ed. (Freeman, San Francisco, 1980), pp. 121-225.

[2] L. D. Landau and E. M. Lifshitz, Statistical Physics, 3rd ed., Part 1 (Elsevier, New York, 1980), pp. 158-183.

[3] R. K. Pathria and P. D. Beale, Statistical Mechanics, 3rd ed. (Elsevier, New York, 2011), p. 148.

[4] D. J. Bedingham, Bose-Einstein condensation in the canonical ensemble, Phys. Rev. D 68, 105007 (2003).

[5] W. J. Mullin and J. P. Fernández, Bose-Einstein condensation, fluctuations, and recurrence relations in statistical mechanics, Am. J. Phys. 71, 661 (2003).

[6] A. N. Wenz, G. Zürn, S. Murmann, I. Brouzos, T. Lompe, and S. Jochim, From few to many: Observing the formation of a Fermi sea one atom at a time, Science 342, 457 (2013).

[7] M. F. Parsons, F. Huber, A. Mazurenko, C. S. Chiu, W. Setiawan, K. Wooley-Brown, S. Blatt, and M. Greiner, SiteResolved Imaging of Fermionic ${ }^{6} \mathrm{Li}$ in an Optical Lattice, Phys. Rev. Lett. 114, 213002 (2015).

[8] L. W. Cheuk, M. A. Nichols, M. Okan, T. Gersdorf, V. V. Ramasesh, W. S. Bakr, T. Lompe, and M. W. Zwierlein, Quantum-Gas Microscope for Fermionic Atoms, Phys. Rev. Lett. 114, 193001 (2015).

[9] E. Haller, J. Hudson, A. Kelly, D. A. Cotta, B. Peaudecerf, G. D. Bruce, and S. Kuhr, Single-atom imaging of fermions in a quantum-gas microscope, Nat. Phys. 11, 738 (2015).

[10] S. Pegahan, J. Kangara, I. Arakelyan, and J. E. Thomas, Spin-energy correlation in degenerate weakly interacting Fermi gases, Phys. Rev. A 99, 063620 (2019).

[11] B. Mukherjee, Z. Yan, P. B. Patel, Z. Hadzibabic, T. Yefsah, J. Struck, and M. W. Zwierlein, Homogeneous Atomic Fermi Gases, Phys. Rev. Lett. 118, 123401 (2017).

[12] K. Hueck, N. Luick, L. Sobirey, J. Siegl, T. Lompe, and H. Moritz, Two-Dimensional Homogeneous Fermi Gases, Phys. Rev. Lett. 120, 060402 (2018).

[13] B. Mukherjee, P. B. Patel, Z. Yan, R. J. Fletcher, J. Struck, and M. W. Zwierlein, Spectral Response and Contact of the Unitary Fermi Gas, Phys. Rev. Lett. 122, 203402 (2019).

[14] R. Onofrio, Cooling and thermometry of atomic Fermi gases, Phys. Usp. 59, 1129 (2016).

[15] G. A. Phelps, A. Hébert, A. Krahn, S. Dickerson, F. Öztürk, S. Ebadi, L. Su, and M. Greiner, Sub-second production of a quantum degenerate gas, arXiv:2007.10807 (2020).

[16] S. Giorgini, L. P. Pitaevskii, and S. Stringari, Theory of ultracold atomic Fermi gases, Rev. Mod. Phys. 80, 1215 (2008).

[17] M. Horodecki, P. Horodecki, and R. Horodecki, Limits for Entanglement Measures, Phys. Rev. Lett. 84, 2014 (2000). where

$$
Y_{B, 1 \leqslant m \leqslant \ell-1}=\frac{(-1)^{m+1}}{Z_{B, N}} \sum_{k=m-1}^{\ell-2}(-1)^{k} Z_{F, k-m+1}\left(\mathcal{S}_{\ell}\right) Z_{B, N-k-1}^{\cup \mathcal{S}_{\ell}},
$$

$Y_{B, 0}=(-1)^{\ell-1}\left\langle n_{j_{1}} n_{j_{2}} \ldots n_{j_{\ell}}\right\rangle_{B, N}, A_{0}=1, A_{0<m<r}=0$, and $A_{r \leqslant m \leqslant \ell-1}\left(\mathcal{S}_{r}\right)=(-1)^{r-1} e^{-\beta \sum_{i_{v} \in \mathcal{S}_{r}} \epsilon_{i_{\nu}}} Z_{B, m-r}\left(\mathcal{S}_{r}\right)$. Also, the term $b_{B}\left(\mathcal{S}_{r}\right)=(-1)^{r-1}\left\langle n_{i_{1}} n_{i_{2}} \ldots n_{i_{r}}\right\rangle_{B, N}$.
[18] S. D. Bartlett and H. M. Wiseman, Entanglement Constrained by Superselection Rules, Phys. Rev. Lett. 91, 097903 (2003).

[19] H. M. Wiseman and J. A. Vaccaro, Entanglement of Indistinguishable Particles Shared Between Two Parties, Phys. Rev. Lett. 91, 097902 (2003)

[20] H. M. Wiseman, S. D. Bartlett, and J. A. Vaccaro, Ferreting out the fluffy bunnies: Entanglement constrained by generalized superselection rules, in Laser Spectroscopy (World Scientific, Singapore, 2004), pp. 307-314.

[21] J. A. Vaccaro, F. Anselmi, and H. M. Wiseman, Entanglement of identical particles and reference phase uncertainty, Int. J. Quantum. Inform. 01, 427 (2003).

[22] N. Schuch, F. Verstraete, and J. I. Cirac, Nonlocal Resources in the Presence of Superselection Rules, Phys. Rev. Lett. 92, 087904 (2004).

[23] J. Dunningham, A. Rau, and K. Burnett, From pedigree cats to fluffy-bunnies, Science 307, 872 (2005).

[24] M. Cramer, M. B. Plenio, and H. Wunderlich, Measuring Entanglement in Condensed Matter Systems, Phys. Rev. Lett. 106, 020401 (2011).

[25] I. Klich and L. S. Levitov, Scaling of entanglement entropy and superselection rules, arXiv:0812.0006 (2008).

[26] S. Murciano, P. Ruggiero, and P. Calabrese, Symmetry resolved entanglement in two-dimensional systems via dimensional reduction, J. Stat. Mech: Theory Exp. (2020) 083102.

[27] M. T. Tan and S. Ryu, Particle number fluctuations, Rényi entropy, and symmetry-resolved entanglement entropy in a twodimensional Fermi gas from multidimensional bosonization, Phys. Rev. B 101, 235169 (2020).

[28] S. Murciano, G. D. Giulio, and P. Calabrese, Entanglement and symmetry resolution in two dimensional free quantum field theories, J. High Energ. Phys. 8 (2020) 073.

[29] L. Capizzi, P. Ruggiero, and P. Calabrese, Symmetry resolved entanglement entropy of excited states in a CFT, J. Stat. Mech.: Theory Exp. (2020) 073101.

[30] S. Fraenkel and M. Goldstein, Symmetry resolved entanglement: Exact results in 1D and beyond, J. Stat. Mech.: Theory Exp. (2020) 033106.

[31] N. Feldman and M. Goldstein, Dynamics of charge-resolved entanglement after a local quench, Phys. Rev. B 100, 235146 (2019).

[32] H. Barghathi, E. Casiano-Diaz, and A. Del Maestro, Operationally accessible entanglement of one-dimensional spinless fermions, Phys. Rev. A 100, 022324 (2019).

[33] R. Bonsignori, P. Ruggiero, and P. Calabrese, Symmetry resolved entanglement in free fermionic systems, J. Phys. A: Math. Theor. 52, 475302 (2019). 
[34] H. Barghathi, C. M. Herdman, and A. Del Maestro, Rényi Generalization of the Accessible Entanglement Entropy, Phys. Rev. Lett. 121, 150501 (2018).

[35] M. Kiefer-Emmanouilidis, R. Unanyan, J. Sirker, and M. Fleischhauer, Bounds on the entanglement entropy by the number entropy in noninteracting fermionic systems, SciPost Phys. 8, 83 (2020).

[36] S. Murciano, G. D. Giulio, and P. Calabrese, Symmetry resolved entanglement in gapped integrable systems: a corner transfer matrix approach, SciPost Phys. 8, 46 (2020).

[37] M. Goldstein and E. Sela, Symmetry-Resolved Entanglement in Many-Body Systems, Phys. Rev. Lett. 120, 200602 (2018).

[38] H. Sato, Nucleus as a canonical ensemble: Entropy and level density at low temperature, Phys. Rev. C 36, 785 (1987).

[39] S. Cheng and S. Pratt, Isospin fluctuations from a thermally equilibrated hadron gas, Phys. Rev. C 67, 044904 (2003).

[40] S. Pratt and J. Ruppert, Quark-gluon plasma in a finite volume, Phys. Rev. C 68, 024904 (2003).

[41] V. D. Toneev and A. S. Parvan, Canonical strangeness and distillation effects in hadron production, J. Phys. G: Nucl. Partic. 31, 583 (2005).

[42] S. V. Akkelin and Y. M. Sinyukov, Quantum canonical ensemble and correlation femtoscopy at fixed multiplicities, Phys. Rev. C 94, 014908 (2016).

[43] A. Parvan, V. Toneev, and M. Płoszajczak, Quantum statistical model of nuclear multifragmentation in the canonical ensemble method, Nucl. Phys. A 676, 409 (2000).

[44] C. Das, S. Das Gupta, W. Lynch, A. Mekjian, and M. Tsang, The thermodynamic model for nuclear multifragmentation, Phys. Rep. 406, 1 (2005).

[45] B. K. Jennings and S. Das Gupta, Canonical partition function in nuclear physics, Phys. Rev. C 62, 014901 (2000).

[46] R. Rossignoli, Canonical and grand-canonical partition functions and level densities, Phys. Rev. C 51, 1772 (1995).

[47] N. Canosa, R. Rossignoli, and P. Ring, Canonical treatment of fluctuations and random phase approximation correlations at finite temperature, Phys. Rev. C 59, 185 (1999).

[48] R. Rossignoli, N. Canosa, and J. Egido, Finite temperature canonical treatments in the static path approximation, Nucl. Phys. A 605, 1 (1996).

[49] K. K. Gudima, A. S. Parvan, M. Płoszajczak, and V. D. Toneev, Nuclear Multifragmentation in Nonextensive Statistics: Canonical Formulation, Phys. Rev. Lett. 85, 4691 (2000).

[50] V. Vovchenko, B. Dönigus, and H. Stoecker, Canonical statistical model analysis of $p-p, p-\mathrm{pb}$, and $\mathrm{pb}-\mathrm{pb}$ collisions at energies available at the CERN Large Hadron Collider, Phys. Rev. C 100, 054906 (2019).

[51] V. Vovchenko, B. Dönigus, and H. Stoecker, Multiplicity dependence of light nuclei production at LHC energies in the canonical statistical model, Phys. Lett. B 785, 171 (2018).

[52] L. Y. Jia and C. Qi, Generalized-seniority pattern and thermal properties in even Sn isotopes, Phys. Rev. C 94, 044312 (2016).

[53] V. V. Begun, M. I. Gorenstein, and O. S. Zozulya, Fluctuations in the canonical ensemble, Phys. Rev. C 72, 014902 (2005).

[54] J.-H. Fu, Higher moments of multiplicity fluctuations in a hadron-resonance gas with exact conservation laws, Phys. Rev. C 96, 034905 (2017).
[55] P. Garg, D. K. Mishra, P. K. Netrakanti, and A. K. Mohanty, Multiplicity fluctuations in heavy-ion collisions using canonical and grand-canonical ensemble, Eur. Phys. J. A 52, 27 (2016).

[56] S. Acharya et al. (ALICE Collaboration), Production of (anti-) ${ }^{3} \mathrm{He}$ and (anti-) ${ }^{3} \mathrm{H}$ in $p$ - $\mathrm{Pb}$ collisions at $\sqrt{s_{N N}}=5.02$ TeV, Phys. Rev. C 101, 044906 (2020).

[57] H. Schmidt, A simple derivation of distribution functions for Bose and Fermi statistics, Am. J. Phys. 57, 1150 (1989).

[58] P. Borrmann and G. Franke, Recursion formulas for quantum statistical partition functions, J. Chem. Phys. 98, 2484 (1993).

[59] P. Borrmann, J. Harting, O. Mülken, and E. R. Hilf, Calculation of thermodynamic properties of finite Bose-Einstein systems, Phys. Rev. A 60, 1519 (1999).

[60] S. Pratt, Canonical and Microcanonical Calculations for Fermi Systems, Phys. Rev. Lett. 84, 4255 (2000).

[61] C. Weiss and M. Wilkens, Particle number counting statistics in ideal Bose gases, Opt. Express 1, 272 (1997).

[62] J. Arnaud, J. M. Boé, L. Chusseau, and F. Philippe, Illustration of the Fermi-Dirac statistics, Am. J. Phys. 67, 215 (1999).

[63] K. Schönhammer, Deviations from Wick's theorem in the canonical ensemble, Phys. Rev. A 96, 012102 (2017).

[64] O. Giraud, A. Grabsch, and C. Texier, Correlations of occupation numbers in the canonical ensemble and application to a Bose-Einstein condensate in a one-dimensional harmonic trap, Phys. Rev. A 97, 053615 (2018).

[65] K. Tsutsui and T. Kita, Quantum correlations of ideal Bose and Fermi gases in the canonical ensemble, J. Phys. Soc. Jpn. 85, 114603 (2016).

[66] C.-C. Zhou and W.-S. Dai, Canonical partition functions: ideal quantum gases, interacting classical gases, and interacting quantum gases, J. Stat. Mech.: Theory Exp. (2018) 023105.

[67] D. S. Dean, P. Le Doussal, S. N. Majumdar, and G. Schehr, Noninteracting fermions at finite temperature in a $d$ dimensional trap: Universal correlations, Phys. Rev. A 94, 063622 (2016).

[68] R. Denton, B. Mühlschlegel, and D. J. Scalapino, Electronic Heat Capacity and Susceptibility of Small Metal Particles, Phys. Rev. Lett. 26, 707 (1971).

[69] R. Denton, B. Mühlschlegel, and D. J. Scalapino, Thermodynamic properties of electrons in small metal particles, Phys. Rev. B 7, 3589 (1973).

[70] K. Schönhammer and V. Meden, Fermion-boson transmutation and comparison of statistical ensembles in one dimension, Am. J. Phys. 64, 1168 (1996).

[71] A. Svidzinsky, M. Kim, G. Agarwal, and M. O. Scully, Canonical ensemble ground state and correlation entropy of Bose-Einstein condensate, New J. Phys. 20, 013002 (2018).

[72] P. K. Jha and S. Hirata, Finite-temperature many-body perturbation theory in the canonical ensemble, Phys. Rev. E 101, 022106 (2020).

[73] K. C. Chase, A. Z. Mekjian, and L. Zamick, Canonical and microcanonical ensemble approaches to Bose-Einstein condensation: The thermodynamics of particles in harmonic traps, Eur. Phys. J. B 8, 281 (1999).

[74] K. Schönhammer, Thermodynamics and occupation numbers of a Fermi gas in the canonical ensemble, Am. J. Phys. 68, 1032 (2000).

[75] V. V. Kocharovsky and V. V. Kocharovsky, Analytical theory of mesoscopic Bose-Einstein condensation in an ideal gas, Phys. Rev. A 81, 033615 (2010). 
[76] J.-H. Wang and Y.-L. Ma, Thermodynamics and finite-size scaling of homogeneous weakly interacting Bose gases within an exact canonical statistics, Phys. Rev. A 79, 033604 (2009).

[77] W. Magnus, L. Lemmens, and F. Brosens, Quantum canonical ensemble: A projection operator approach, Physica A 482, 1 (2017).

[78] A. Grabsch, S. N. Majumdar, G. Schehr, and C. Texier, Fluctuations of observables for free fermions in a harmonic trap at finite temperature, SciPost Phys. 4, 14 (2018).

[79] M. Kruk, M. Łebek, and K. Rzążewski, Statistical properties of cold bosons in a ring trap, Phys. Rev. A 101, 023622 (2020).

[80] J. Grela, S. N. Majumdar, and G. Schehr, Kinetic Energy of a Trapped Fermi Gas at Finite Temperature, Phys. Rev. Lett. 119, 130601 (2017).

[81] K. Liechty and D. Wang, Asymptotics of free fermions in a quadratic well at finite temperature and the Moshe-NeubergerShapiro random matrix model, Ann. Inst. Henri Poincaré Probab. Statist. 56, 1072 (2020).
[82] G. C. Wick, The Evaluation of the Collision Matrix, Phys. Rev. 80, 268 (1950).

[83] Here we replace $Z_{B, x}$ with $Z_{B, x}^{\cup\{j\}}$ and $Z_{B, x}^{\backslash\{j\}}$ with $Z_{B, x}$ in (16) and (14).

[84] All code, scripts and data used in this work are included in a GitHub repository: https://github.com/DelMaestroGroup/ papers-code-CanonicalEnsembleTheory. Permanent link: https://doi.org/10.5281/zenodo.3970773.

[85] H.-J. Schmidt and J. Schnack, Thermodynamic fermion-boson symmetry in harmonic oscillator potentials, Physica A 265, 584 (1999).

[86] I. Peschel, Calculation of reduced density matrices from correlation functions, J. Phys. A: Math. Gen. 36, L205 (2003).

[87] I. Peschel and V. Eisler, Reduced density matrices and entanglement entropy in free lattice models, J. Phys. A: Math. Theor. 42, 504003 (2009).

[88] V. Eisler and I. Peschel, Analytical results for the entanglement hamiltonian of a free-fermion chain, J. Phys. A: Math. Theor. 50, 284003 (2017)

[89] I. Peschel, Special review: Entanglement in solvable manyparticle models, Braz. J. Phys. 42, 267 (2012). 